archives-ouvertes

\title{
Imaging non-Brownian particle suspensions with X-ray tomography: application to the microstructure of Newtonian and visco-plastic suspensions
}

\author{
Stephanie Deboeuf, Nicolas Lenoir, David Hautemayou, Michel Bornert, \\ Guillaume Ovarlez
}

\section{To cite this version:}

Stephanie Deboeuf, Nicolas Lenoir, David Hautemayou, Michel Bornert, Guillaume Ovarlez. Imaging non-Brownian particle suspensions with X-ray tomography: application to the microstructure of Newtonian and visco-plastic suspensions. Journal of Rheology, 2017. hal-02171334

\section{HAL Id: hal-02171334 \\ https://hal-enpc.archives-ouvertes.fr/hal-02171334}

Submitted on 10 Jul 2019

HAL is a multi-disciplinary open access archive for the deposit and dissemination of scientific research documents, whether they are published or not. The documents may come from teaching and research institutions in France or abroad, or from public or private research centers.
L'archive ouverte pluridisciplinaire HAL, est destinée au dépôt et à la diffusion de documents scientifiques de niveau recherche, publiés ou non, émanant des établissements d'enseignement et de recherche français ou étrangers, des laboratoires publics ou privés. 


\title{
Imaging non-Brownian particle suspensions with X-ray tomography:
}

\section{application to the microstructure of Newtonian and visco-plastic suspensions}

\author{
S. Deboeuf* \\ Sorbonne Universités, UPMC Univ. Paris 06, CNRS, UMR 7190, \\ Institut Jean Le Rond dAlembert, 75005 Paris, France \\ N. Lenoir \\ PLACAMAT, UMS 3626 CNRS-Univ. de Bordeaux, Pessac, France \\ D. Hautemayou and M. Bornert \\ Université Paris-Est, Laboratoire Navier (UMR 8205) CNRS, \\ ENPC, IFSTTAR, F-77420 Marne-la-Vallée, France \\ F. Blanc \\ CNRS-University of Nice, LPMC-UMR 7336, 06108 Nice, France \\ G. Ovarlez \\ Univ. Bordeaux, CNRS, Solvay, LOF, \\ UMR 5258, F-33608 Pessac, France
}

(Dated: September 29, 2017) 


\begin{abstract}
A key element in the understanding of the rheological behaviour of suspensions is their microstructure. Indeed, the spatial distribution of particles is known to depend on flow history in suspensions, which has an impact on their macroscopic properties. These micro-macro couplings appeal for the development of experimental tools allowing for the rheological characterization of a suspension and the imaging of particles. In this paper, we present the technique we developed to image in three dimensions the microstructure of suspensions of non-Brownian particles, using X-ray computed tomography and sub-voxel identification of particle centers. We also give examples of the information we can get in the case of Newtonian and visco-plastic suspensions, referring to Newtonian and visco-plastic suspending fluid. We compute three dimensional pair distribution functions and show that it is possible to get a nearly isotropic microstructure after mixing. Under shear, this microstructure becomes anisotropic in the shear plane (velocity-velocity gradient plane), whereas it is almost isotropic in the two other planes (velocity-vorticity and velocity gradient-vorticity planes). When changing the plane of shear (from a squeeze flow to a rotation flow), the microstructure reorganizes to follow this change of shear plane. It is known that for Newtonian suspensions the anisotropy is independent on the shear rate; we show here that for a visco-plastic suspension it depends on it. Finally, we study the structuration close to boundaries and we evidence some particle alignment along both solid surfaces and, more surprisingly, along free interfaces.
\end{abstract}

\footnotetext{
*sdeboeuf@dalembert.upmc.fr; http://www.ida.upmc.fr/ deboeuf/
} 


\section{${ }_{19}$ I. INTRODUCTION}

20 Many flowing materials encountered in nature (landslides, debris or lava flows, ...) or ${ }_{21} \mathrm{raw}$ materials produced in industry (concrete, batter, ...) are mixtures of dispersed solids in 22 a liquid. These systems are composed of a wide range of materials of various sizes, which 23 makes them complex to understand. In order to predict the behaviour and optimize the use 24 of these suspensions, a useful step is to identify model systems capable of reproducing the ${ }_{25}$ phenomenology of interest and to understand the rheology of controlled suspensions. When ${ }_{26}$ length scales between the coarser and the finer dispersed particles of a suspension are well ${ }_{27}$ separated, a possible model system is a visco-plastic suspension of solid particles, referring to 28 a visco-plastic suspending fluid, i.e. of nonlinear viscosity with a yield stress. Indeed, from a 29 rheological point of view, a fresh concrete can be considered as coarse particles (sand, gravel) 30 suspended in a visco-plastic fluid (cement paste) [1, 2]. Even more simply, one can consider 31 a Newtonian suspension of solid particles as a model system, referring to a Newtonian 32 suspending fluid [3]. A lot can indeed be learnt from such materials: studying first the 33 interactions of multiple particles in a linear (Newtonian) fluid is crucial to understand their 34 interaction in a nonlinear medium $[4,5]$. This knowledge can then be applied to materials 35 closer to applications such as cementitious materials $[2,6]$.

36 A key element in the understanding of the rheological behaviour of suspensions is their 37 microstructure $[7,8]$. Indeed, the spatial distribution of particles in suspensions is known to 38 depend on the flow history - an anisotropic distribution is induced by shear -, which has an 39 impact on their properties [7, 9-14] - an anisotropic microstructure is at the origin of normal 40 stress differences and of viscosity variations during transient regimes. These micro-macro ${ }_{41}$ couplings between microstructure and flow appeal for the development of experimental and 42 numerical tools allowing for the characterization of the rheology of a suspension and the ${ }_{43}$ imaging of its microstructure. A lot of numerical studies used Stokesian dynamics equations ${ }_{44}[8,15-18]$. The existing experimental techniques efficient for non-Brownian suspensions are 45 all based on optics. Non-Brownian particles are indeed too large to use scattering techniques 46 such as X-ray scattering or coherent diffraction imaging. For non transparent particles in a ${ }_{47}$ transparent fluid, it is nevertheless possible to image the microstructure only near the free 48 surface [10] or near a transparent boundary. As we will demonstrate here, the proximity of a 49 boundary (a solid wall or a free interface) influences strongly the microstructure and makes 
50 it different from the one in the bulk. The bulk microstructure can be obtained only for ${ }_{51}$ specific suspensions, where particles and the fluid have the same optical index; a laser sheet ${ }_{52}$ can then be used to light a plane embedding a fluorescent stain so as to visualize and identify ${ }_{53}$ particles in two dimensions (2D) [11, 12]. Obtaining a three dimensional microstructure ${ }_{54}$ then implies to displace the position of the laser sheet. Confocal microscopy can also be used ${ }_{55}$ for micron-sized particles $[18,19]$, submitted to Brownian diffusion, that is in competition 56 with shear flow, quantified by the Peclet number (being the ratio of hydrodynamic over ${ }_{57}$ thermal forces). Here we choose to use non intrusive X-ray techniques as a promising tool ${ }_{58}$ to image in the bulk suspensions in three dimensions (3D).

59 In this paper, we present the technique we developed to image in 3D the microstructure 60 of suspensions of non-Brownian particles, using X-ray computed tomography (CT) and then ${ }_{61}$ identifying by image analysis the particle centers with a sub-voxel resolution. All the details 62 to duplicate our technique are given. We give examples of the information we can get in the ${ }_{63}$ case of Newtonian and visco-plastic suspensions. We compute 3D pair distribution functions ${ }_{64}$ (pdf) and show that it is possible to get a nearly isotropic microstructure after mixing. Under 65 shear, this microstructure becomes anisotropic in the shear plane (velocity-velocity gradient ${ }_{66}$ plane), whereas it is nearly isotropic in the two other planes (velocity-vorticity and velocity ${ }_{67}$ gradient-vorticity planes). When changing the plane of shear (from a squeeze flow to a ${ }_{68}$ rotation flow), the microstructure reorganizes to follow this change of shear plane. While it ${ }_{69}$ is known that for Newtonian suspensions the anisotropy is independent on the shear rate, we 70 show that for a visco-plastic suspension it depends on it. Finally, we study the structuration ${ }_{71}$ close to boundaries and we evidence particle alignment along both solid surfaces and more 72 surprisingly along free interfaces.

73 In Sec II, we present the principles of our study and we provide the details on the studied 74 suspensions and the set-up. Sec III is devoted to the X-ray tomography technique and to the 75 method we developed to identify particles center and compute pair distribution functions. 76 In Sec IV, we present various examples of the microstructure of Newtonian and visco-plastic 77 suspensions. 
a)

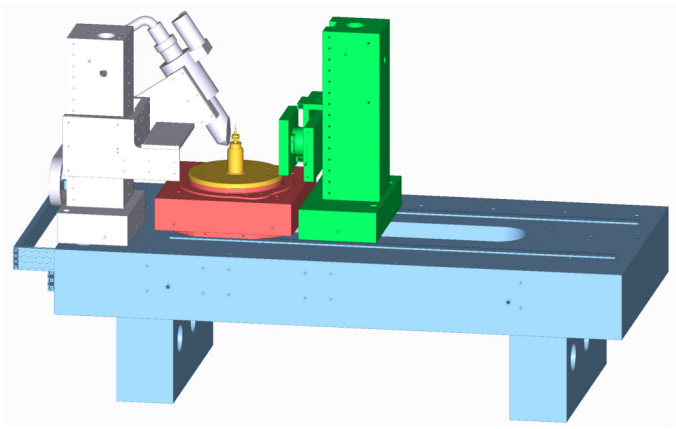

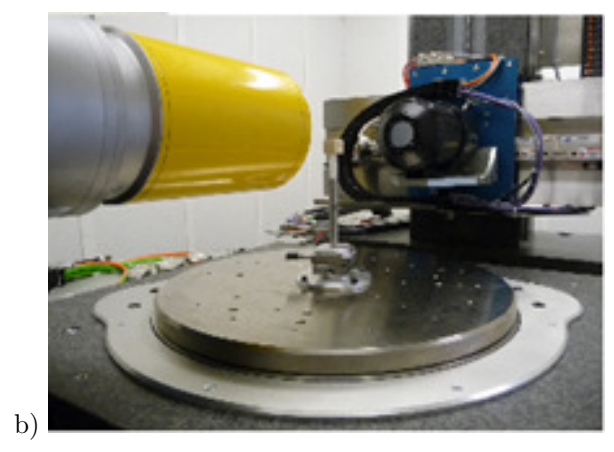

FIG. 1. X-ray computed micro-tomography set-up (a and b) and dedicated parallel plates set-up (c). The sketch (a) shows the X-ray source (in white), the imaging device (in green), the rotation (in red) and translation (in blue) stages and the parallel plates set-up (in yellow).

\section{MATERIALS AND METHODS}

79

80 81 of micro-mechanical models for complex suspensions rheology. Thus, we need to image 82 particles in a flow cell and to characterize their spatial distribution for various flow histories.

83 Our system made of a suspension in a rheometrical set-up should allow for the investiga${ }_{84}$ tion from small to large space scales, from the length of the dispersed particles to the whole 85 suspension. The flow cell should be large enough to be representative of a macroscopic shear 86 flow, to ensure that the material exhibits a bulk behaviour not influenced by finite size or 87 non local effects [20], and to get enough statistics for studying the microstructure. This ${ }_{88}$ leads us to choose a rheometer equipped with a parallel plates geometry (Fig. 1c). In the ${ }_{89}$ same time as doing rheology, we want to image the microstructure in $3 \mathrm{D}$, even in the case 90 of non matched optical index materials. This leads us to use X-ray CT imaging, which is ${ }_{91}$ efficient for many suspensions and geometries, as long as there is enough X-ray absorption 92 contrast between particles and the fluid.

93 The sample to image is placed on a rotating stage between an X-ray source and a 2D 94 imaging device (Fig. 1), which monitors the transmission of X-rays through the sample. In 95 our case, the imaging device was a PaxScan2520V flat panel detector from Varian, made of 96 a CSI scintillator and a matrix of 1920x1536 pixels (among which 1840x1456 are effective), 97 with a pixel width of $127 \mu \mathrm{m}$. This provides a $2 \mathrm{D}$ radiograph, accounting for the total 
98 absorption along linear paths through the sample. A series of 2D radiographs for different 99 rotation angles of the sample (typically over $360^{\circ}$ in a lab X-ray CT device) then allows for 100 the reconstruction of the 3D map of absorption coefficients [21-23]. If particles and the fluid 101 do not have the same absorption coefficient, we can finally achieve to detect the particles. 102 This map is discretized over elementary volumes called voxels (i.e. volumic pixels). The 103 achievable minimum voxel size is given by the ratio of the size of the imaged sample over ${ }_{104}$ the number of pixels in the detector width. The typical width of the CCD detectors used 105 for X-ray CT is about 2000 pixels. Noting $D$, the diameter of the parallel plates geometry, 106 the minimum possible voxel size is $D / 2000$. To identify precisely the particles center, their ${ }_{107}$ diameter $d$ should be at least 10 voxels, i.e. $d \gtrsim D / 200$. The gap size $H$ between the plates 108 in the parallel plates geometry should contain at least 10 particles to avoid some finite size 109 effects, i.e. $H \gtrsim D / 20$, which is compatible with the study here but limits the possible 110 aspect ratios diameter/gap of the geometry to $D / H \lesssim 20$. We will show later that our 111 home-made algorithms allow us to refine particle centers positions at a sub-voxel resolution 112 of an order of $d / 100$, that ultimately allows for a fine characterization of the microstructure. ${ }_{113}$ Here (Fig. 2a), the diameter of the parallel plates geometry id $D=2 \mathrm{~cm}$, the gap of the ${ }_{114}$ geometry is $H=2 \mathrm{~mm}$, the size of particles $d$ is between $100 \mu \mathrm{m}$ and $200 \mu \mathrm{m}$, the size of 115 voxels is $12 \mu \mathrm{m}$ and the final accuracy on the particles centers in most cases presented here 116 is $6 \mu \mathrm{m}$, but it can be decreased down to $1 \mu \mathrm{m}$ if necessary.

${ }_{117}$ X-ray CT imaging may demand a non negligible time for scanning. The time for one scan 118 depends of the X-ray set-up, the suspension properties and the chosen spatial resolution (X119 ray flux, number of radiographs, time exposure, ...). With our X-ray CT device and the ${ }_{120}$ chosen spatial resolution, the whole sample can be scanned in 20 minutes but with a poor ${ }_{121}$ signal-to-noise ratio. The results presented here are obtained from 1 hour-scan in order to 122 enhance the image quality. During the time of a scan, particles must not move within the ${ }_{123}$ suspending fluid (i.e. displacement less than 1 voxel or even less, if subvoxel accuracy is 124 expected). This leads us to use first a visco-plastic fluid as a suspending fluid ensuring that ${ }_{125}$ particles do not move as long as stresses are smaller than the yield stress. Then we apply to ${ }_{126}$ our suspension a given shear history, then stop the flow and image the material at rest with ${ }_{127}$ a frozen structure, which is supposed to be close to the structure under flow. The underlying 128 hypothesis is that relaxation of the microstructure at rest is negligible. This flow-arresting ${ }_{129}$ technique was already used before for Brownian suspensions [18]. In a second time, we 


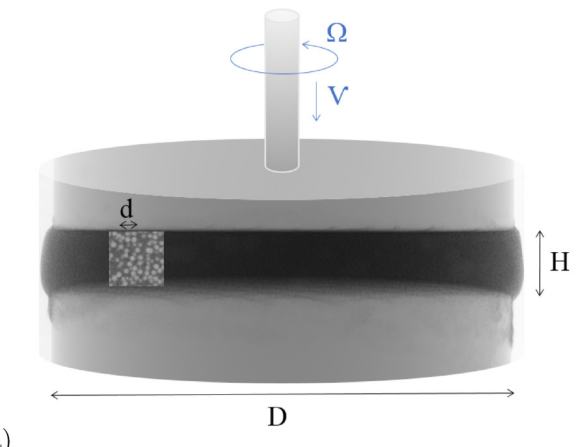

a)

b)
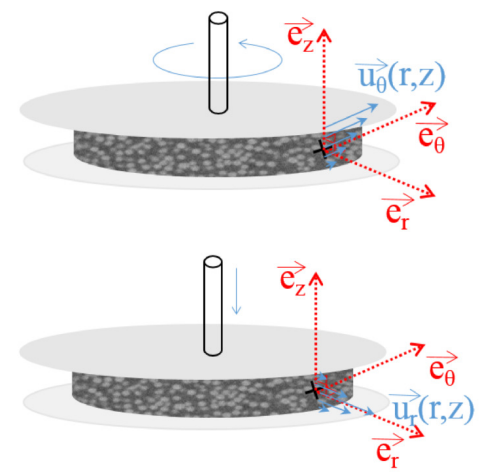

c)

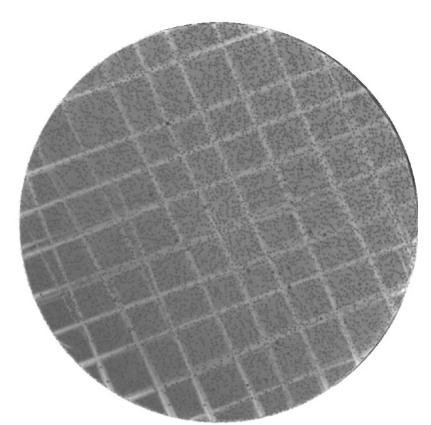

FIG. 2. (a) Scheme of the parallel plates set-up: $D$ and $H$ are the plates diameter and gap, $d$ is the particles diameter, $\Omega$ and $\vartheta$ are the rotational and translational velocities of the top plate. (b) Schemes of the rotational and squeeze shear flows: the suspension velocity $u_{\theta}(r, z)$ and $u_{r}(r, z)$ are aligned with the azimuthal and radial directions respectively. (c) A picture processed from X-ray slices showing the rough surface with grooves of the set-up's plates.

130 succeed to use a Newtonian suspension with density-matched particles and fluid, to avoid 131 any buoyancy effects at rest. In principe, various types of suspensions may be imaged and 132 studied with our technique, as soon as particles or suspended objects do not move during the 133 time of a X-ray scan. For particles as small as a few $\mu m$, Brownian fluctuations may call for ${ }_{134}$ even quicker X-ray micro-tomograph (e.g. Synchrotron source). In terms of concentration 135 of particles, we have successfully image in 3D suspensions at solid fractions as high as 50\% 136 and it should be possible to go even higher.

TABLE I. Properties of particles and fluids used for the Newtonian and the visco-plastic suspensions: average diameter $d$ of particles, viscosity $\eta$ of the Newtonian fluid, elastic shear modulus $G^{\prime}$, yield stress $\tau_{y}$ and consistency $K$ of the visco-plastic fluid and density $\rho$ of the fluid and density-matched particles.

\begin{tabular}{|c|c|c|} 
PMMA & Newtonian & Density \\
\hline$d(\mu \mathrm{m})$ & $\eta($ Pa.s $)$ & $\rho$ \\
\hline $170 \pm 12$ & 1.3 & 1.19
\end{tabular}

\begin{tabular}{|c|ccc|c|} 
PS & \multicolumn{2}{|c|}{ Yield stress } & Density \\
\hline$d(\mu \mathrm{m})$ & $G^{\prime}(\mathrm{Pa})$ & $\tau_{y}(\mathrm{~Pa})$ & $K\left(\mathrm{~Pa}_{\mathrm{s}} \mathrm{S}^{0.5}\right)$ & $\rho$ \\
\hline $138 \pm 8$ & 250 & 26 & 5.1 & 1.05
\end{tabular}




\section{B. Suspensions of non-Brownian particles}

${ }_{138}$ Two suspensions are studied here (Table I): one is composed of poly(methyl methacry139 late) (PMMA) spheres in a Newtonian fluid and the other is composed of polystyrene 140 (PS) spheres in a visco-plastic fluid. In both cases, particles and suspending fluids are ${ }_{141}$ density-matched to avoid any buoyancy effects. The PMMA particles average diameter 142 is $170 \mu \mathrm{m} \pm 12 \mu \mathrm{m}$ and the PS particles average diameter is $138 \mu \mathrm{m} \pm 8 \mu \mathrm{m}$. These diameters 143 were measured from optical pictures of bead samples. With such a size, particles are non144 Brownian and supposed to be non-colloidal. For all of experiments presented in this work, 145 the solid fraction $\phi$ of the suspensions was observed to be uniform within our rheological 146 set-up.

147 The Newtonian suspending fluid is an acrylic double matching liquid from Cargille Lab. ${ }_{148}$ Its density is 1.19 and its viscosity $1300 \mathrm{mPa} . \mathrm{s}$ at $25^{\circ} \mathrm{C}$. The natural contrast of X-ray 149 absorption between particles and the fluid is sufficient to separate them in final X-ray CT 150 images.

151 The visco-plastic fluid, of density 1.05, is a concentrated emulsion of aqueous drops 152 dispersed at a $77.5 \%$ volume fraction (volume of dispersed phase over total volume) in a 153 continuous oily phase. Surfactant SPAN80 is added in dodecane oil at $7.5 \%$ in mass (SPAN80 154 mass over dodecane mass) and iodine sodium salt is added in water at $15 \%$ in mass (NaI 155 mass over $\mathrm{H}_{2} \mathrm{O}$ mass) to stabilize the emulsion and enhance contrast for X-ray imaging. The 156 droplets size is of order of $1 \mu \mathrm{m}$, much smaller than the particles size. This ensures that the 157 emulsion is seen as a continuous material - a visco-plastic fluid - by the particles [2, 4, 5].

${ }_{158}$ The behaviour of the visco-plastic fluid is well described by a Herschel-Bulkley law, relating 159 the shear stress $\tau$ to the shear rate $\dot{\gamma}$, with $\tau_{y}=26 \mathrm{~Pa}$ and $K=5.1 \mathrm{~Pa} . \mathrm{s}^{0.5}$ :

$$
\tau=\tau_{y}+K \dot{\gamma}^{0.5}, \text { if } \tau \geq \tau_{y}
$$

160 valid in the steady flow regime, i.e. for stresses larger than the yield stress $\tau_{y}$. The elastic ${ }_{161}$ contribution to its behaviour is accounted for by the elastic shear modulus $G^{\prime}=250 \mathrm{~Pa}$.

${ }_{162}$ More details on the rheology of both background (Newtonian and visco-plastic) fluids, 163 with and without particles, in [5] and [24] respectively. 


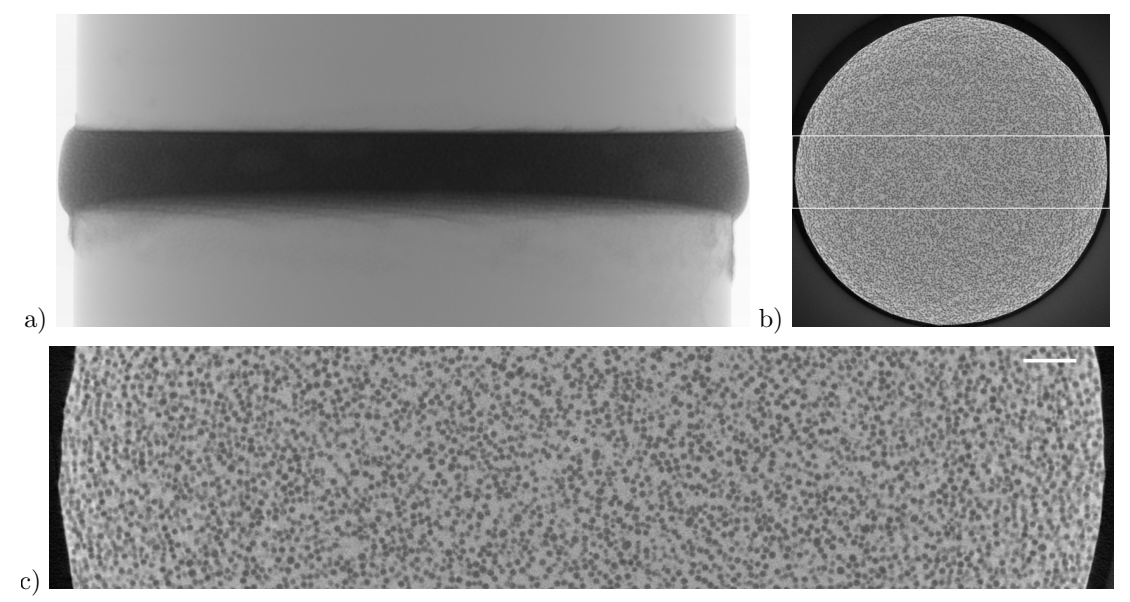

FIG. 3. X-ray radiograph corresponding to the transmitted intensity of X-rays through the sample (the visco-plastic suspension) along one linear path (a), reconstructed horizontal slice encoding for X-ray absorption of voxels in the shear cell at a solid fraction $\phi \simeq 35 \%$ (b) and zoom of this slice (c) corresponding to the white rectangle drawn in (b). The scale bar drawn in (c) is $1 \mathrm{~mm}$ long.

\section{X-ray microtomography}

165

In this study, we used the X-ray microtomography device of Laboratoire Navier, made 166 up of an Ultratom scanner designed and assembled by RX Solutions (Annecy, France) on 167 the basis of the specific requests of Laboratoire Navier. Using the commercial software Xact 168 developed by this company, based on the filtered retroprojection algorithm adapted to cone169 beam geometry [25], 3D images encoding for the X-ray absorption field are reconstructed 170 from the recorded 2D radiographs. Consistently with the definition of the final 3D images, 1711440 radiographs have been recorded over $360^{\circ}$. Note that for each rotation angle, 6 radio172 graphs (with an exposure time for one radiograph of $1 / 3 \mathrm{~s}$ ) have been averaged to improve 173 the signal to noise ratio. The final 3D images had a resolution of $12 \mu \mathrm{m}$ and a definition of 174 1840x1840x170 voxels.

175 One of the advantages of this technique is that the spatial resolution can be isotropic, con176 trary to other scanning techniques, such as confocal microscopy, laser scanning or refractive 177 index matched scanning [12, 26-29]. This allows to get information on the microstructure 178 with the same accuracy in all planes. Figure 3 shows one example of a 2D radiograph of the 179 visco-plastic suspension confined between the two parallel plates and $12 \mu \mathrm{m}$-thick horizontal 180 slices of the reconstructed 3D image for a solid fraction $\phi \simeq 35 \%$. 
The suspension is sheared at the macroscopic scale in a parallel plates geometry (Fig. 2a) 183 of diameter $D=2 \mathrm{~cm}$ and gap $H=2 \mathrm{~mm}$ (aspect ratio $D / H=10$ ), controlled by a rheometer 184 (Kinexus, Malvern). The plates of the shear cell (Fig. 2c) are made rough to prevent slipping 185 of the suspension at solid boundaries. They have been treated as follow: each PMMA plate 186 was roughened thanks to sand-paper of roughness size $150 \mu \mathrm{m}$, then a grid pattern of grooves 187 was imprinted thanks to a file with an edge (triangular lime). In the following, we will 188 compare some results with smooth PMMA plates and roughened PMMA plates.

${ }_{189}$ To image a sample in 3D, 2D radiographs have to be recorded for many different view 190 angles, which means that X-rays have to be allowed to propagate through the sample for 191 all directions nearly perpendicular to the rotation axis, which would not be possible with 192 a standard rheometer (its body would block X-rays when placed between the source and 193 the detector). This leads us to build a dedicated parallel plates set-up, made of PMMA to 194 ensure a low X-ray absorption by the set-up (Fig. 1c). This set-up can be inserted in the ${ }_{195}$ rheometer for shearing the material and characterizing its rheological response. After a given 196 shear history in the rheometer, it can then be blocked thanks to a chuck, removed from the 197 rheometer and carefully moved (to avoid any disturbance) to the X-ray CT set-up, where it 198 is put on the rotating stage for imaging. During the whole duration of X-ray imaging, the 199 parallel plates set-up is blocked. We checked that our ex-situ experiments (moved from the 200 rheometer to the X-ray CT set-up) lead to the same results as in-situ ones (for which both 201 shear and imaging are on the spot).

${ }_{202}$ In a parallel plates geometry, when rotation is imposed to the top plate at a rate $\Omega$ 203 while keeping fixed the bottom plate (Fig. 2b), the force balance equation and boundary 204 conditions (no slip at the two plates) predict a simple shear of the fluid in the orthoradial ${ }_{205}$ plane $(\theta z)$, the single non-zero value of the shear rate tensor being:

$$
\dot{\gamma}(r)=2 d_{z \theta}(r)=\Omega r / H
$$

206 with an azimuthal velocity:

$$
u_{\theta}(r, z)=\Omega z r / H
$$

${ }_{207}(r, \theta, z)$ being the cylindrical coordinates [30]. The rotational shear is not homogeneous but 208 depends linearly on the radial direction $r$. This analysis holds not only for Newtonian fluids, 
209 but for complex fluids too.

${ }_{210}$ In the same geometry of parallel plates, a squeeze flow can be imposed by translating 211 vertically one plate at a velocity $\vartheta$ (Fig. 2b). It can be approximated by a simple shear 212 flow in the radial plane $(r z)$, in the case of a large aspect ratio $D / H$ and of no slip at 213 solid boundaries [30-32]. In this case, the shear rate $\dot{\gamma}(r, z)=2 d_{z r}(r, z)$ is inhomogeneous 214 and a priori unknown: it depends on the rheology of the fluid of interest. Nevertheless, 215 a convenient approximation of the maximal shear rate, valid for a Newtonian fluid in the 216 lubrication approximation, is:

$$
\dot{\gamma}=3 D \vartheta /\left(2 H^{2}\right)
$$

${ }_{217}$ Note that by symmetry, the shear has a different sign in the upper and lower part of the 218 suspension through the central surface at $z=0$.

${ }_{219}$ In order to shear the material in a parallel plates geometry, it is first poured on the 220 bottom plate, then it is squeezed by the top plate to reach the required gap. So even if the 221 interest is in a rotational shear, the material generally experiences first a squeeze flow during 222 the loading of the fluid in the geometry, which might affect its initial state. That is why in 223 the following, we will investigate the impact of both flows on the suspension microstructure.

\section{III. IMAGE ANALYSIS}

225 An accurate identification of particle centers from the obtained 3D images is crucial for the 226 computation of pair vectors and pair distribution functions to characterize the microstructure 227 of the suspension. Thus, we choose to develop home-made algorithms to be able to identify ${ }_{228}$ particles center at a sub-voxel resolution.

229 In all the text, the 3D images $I$ we work with are fields of absorption coefficients encoded 230 as intensity levels without unit measurement. In practice, 16 bits images have been used 231 (grey levels between 0 and $2^{16}-1$ ).

${ }_{232}$ Figure 4 shows the typical greylevel profile of a particle: grey levels of all voxels in the 233 neighborhood of a particle are plotted against the distance $r$ to the voxel considered to be 234 its center. Assuming the density of the particles to be uniform, one would have expected a ${ }_{235}$ Heaviside-like shape for such plots, with a uniform grey level for $r$ lower than the particle 236 radius (of the order of 6 voxels), corresponding to the absorption coefficient of PMMA or ${ }_{237} \mathrm{PS}$, and another (larger) uniform grey level for larger $r$, corresponding to the absorption of 

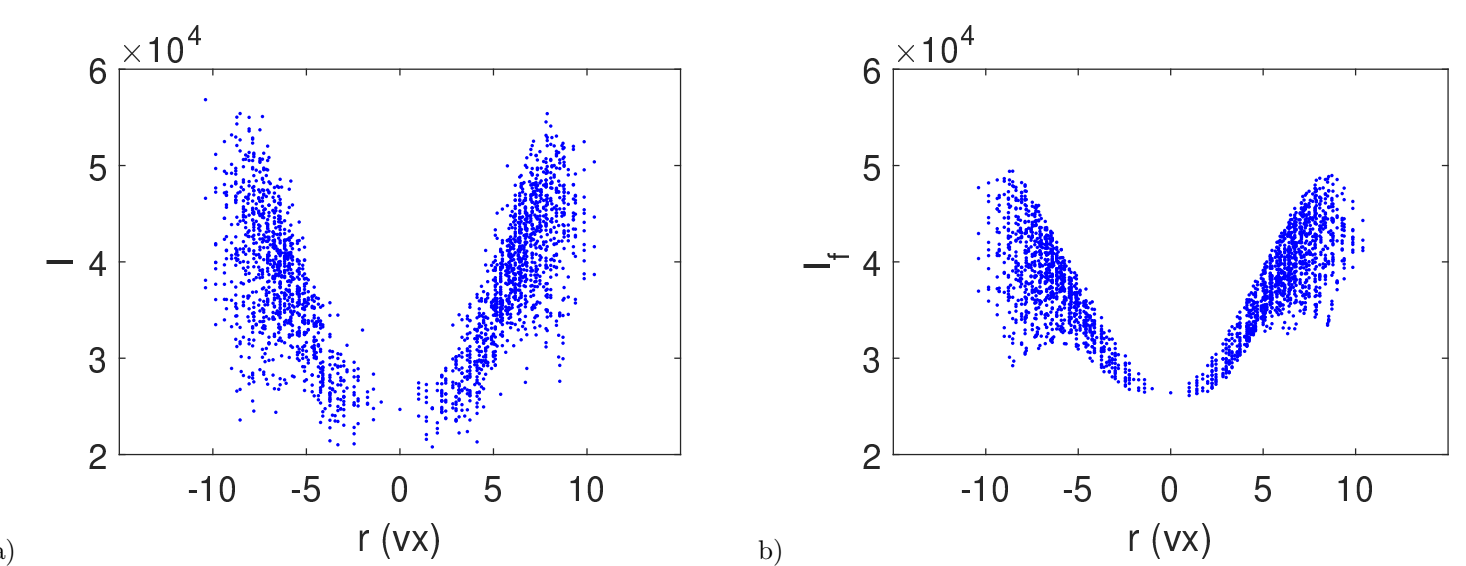

FIG. 4. Absorption levels (without unit measurement, included between 0 and $2^{16}-1$ ) of voxels over one particle and its close neighbourhood in the visco-plastic suspension at a solid fraction $\phi \simeq 40 \%$, as a function of the distance $r$ to its center, from the raw and filtered images $I$ (a) and $I_{f}(\mathrm{~b})$

238 the fluid.

239 Actual profiles first differ from ideal profiles because of natural random image noise, which 240 cannot be avoided, especially if fast imaging conditions are required. Such fluctuations are 241 indeed observed near $r=0$, up to a distance of about 2 to 3 voxels, where grey levels are 242 essentially uniform on average but fluctuate randomly.

243 The second discrepancy with respect to the ideal situation takes the form of a gradual 244 increase of the average grey levels for $r$ varying from about 3 to 7 voxels. While there 245 are still fluctuations associated to natural image noise, the smooth progressive increase of 246 the average grey level is due to the limited spatial resolution of the CT imaging device. ${ }_{247}$ Indeed the grey level of a given voxel in the reconstructed image should be considered as 248 a spatial convolution of the physical absorption coefficient of the material (relative to the 249 spectrum of the polychromatic X-ray beam) with some kernel of small finite size. This 250 kernel characterizes the resolving power of the imaging system. For CT imaging systems, 251 it is sensitive to various parameters, such as the finite spot size of the X-ray source (which ${ }_{252}$ can be evaluated to be about $8 \mu \mathrm{m}$ for our imaging conditions), diffusion effects of the 253 scintillator, fill factor of the pixel matrix, blurring effects of electronic device,... A more ${ }_{254}$ detailed discussion can be found in [33]. In the present setup, and considering the grey level ${ }_{255}$ profile presented in figure 4, the radius of this kernel is likely to be of the order of 2 to 3 
TABLE II. Values of the parameters $\ell_{\text {noise }}, r_{\text {neigh }}, r_{\text {min }}$ and $r_{\text {sym }}$ used for image analyses, with $d$, the diameter of particles

\begin{tabular}{|c|c|c|c|}
$\ell_{\text {noise }}$ & $r_{\text {neigh }}$ & $r_{\text {min }}$ & $r_{\text {sym }}$ \\
\hline 1 voxel & $\sim 7$ voxels & $\sim 6$ voxels & $\sim 7$ voxels \\
$\sim \mathrm{d} / 10$ & $0.6 \mathrm{~d}$ & $0.5 \mathrm{~d}$ & $0.6 \mathrm{~d}$ \\
$\sim 12 \mu \mathrm{m}$ & $\sim 83 \mu \mathrm{m}$ & $\sim 69 \mu \mathrm{m}$ & $\sim 83 \mu \mathrm{m}$
\end{tabular}

256 voxels, which explains that the sharp theoretical particle-matrix interface is degraded into 257 a smooth interface with a thickness of about 5 voxels.

258 A third discrepancy is for $r$ larger than 7 voxels, where the profile seems to tend on 259 average to a uniform grey level, expected to be related to the absorption coefficient of the 260 fluid, but with much larger fluctuations than within the particles. This might be due to 261 other neighbor particles: the voxels that are close to the latter will exhibit a lower grey level 262 than those further away.

263 In the following, we will finally take advantage of these (slightly) inhomogeneous grey ${ }_{264}$ level profiles of particles in order to determine accurately their position.

265 Below we describe the successive steps. First, we detect approximately particle centers 266 as the local minimum absorption and we get rid of false and multiple detections. Second, 267 we refine particle center positions at a sub-voxel resolution by using the symmetry of the in268 creasing absorption around the center. Finally, we explain how we compute pair distribution 269 functions.

\section{A. Detection of particles}

271 We process the 3D images with 3D morphological operations within Matlab. We use as 272 few as possible filtering operations and we introduce as few as possible tunable parameters, 273 whose values are not severely chosen, so that our method depends only slightly on the value 274 of parameters and is efficient for various solid fractions without changing the value of any 275 parameter.

First, we smooth the raw 3D image $I$ by filtering high spatial frequency noise with a 
a)

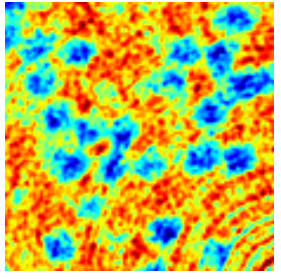

b)

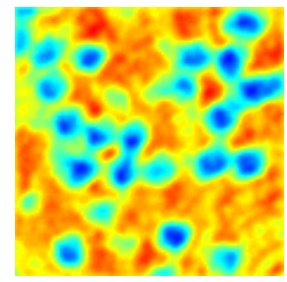

c)

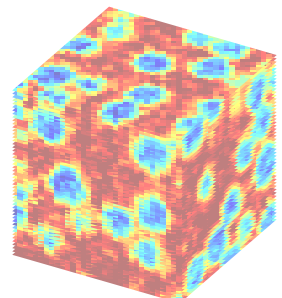

d)

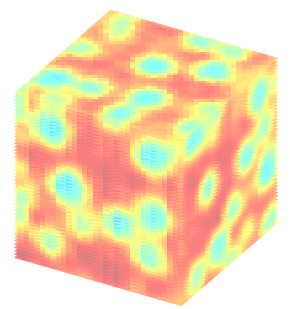

FIG. 5. Two dimensional (a, b) and three dimensional (c, d) images of the visco-plastic suspension of length about $\sim 1 \mathrm{~cm}$ at a solid fraction $\phi \simeq 35 \%$ : raw images $I(\mathrm{a}, \mathrm{c})$ and filtered images $I_{f}(\mathrm{~b}$, d)

${ }_{277}$ Gaussian filter of characteristic size $\ell_{\text {noise }}=1$ voxel: $I_{f}=f * I$ with

$$
f(x)=\exp \left(-\left(\frac{x}{2 \ell_{\text {noise }}}\right)^{2}\right) / \int_{-\infty}^{\infty} \exp \left(-\left(\frac{x}{2 \ell_{\text {noise }}}\right)^{2}\right) d x
$$

278 where the symbol $*$ refers to the convolution operator: $(f * I)\left(x_{0}\right)=\int_{-\infty}^{\infty} f(x) I\left(x-x_{0}\right) d x$, 279 and $I_{f}$ is the image we work with in the following steps (Fig. 4 and 5). This filtering 280 operation has a long-range effect even in the case of a small value for the length $\ell_{\text {noise }}$, due 281 to the extended range of integration in the convolution operation; in practice, the integration 282 is realized over a size of $21 \times 21 \times 21$ voxels, chosen after we checked that a larger size does not 283 change the value of $I_{f}$.

284 Second, we extract the positions of voxels showing a local minimum of absorption as a 285 first estimation of the particle centers.

286 False detections corresponding to local extrema in the air, the plates or the fluid, as well ${ }_{287}$ as multiple detections (several centers for the same particle) are possible. In practice, they 288 represent less than $1 \%$ of all detections; however, we do get rid of these false and multiple 289 detections thanks to the two following steps.

- We first note that X-ray absorption coefficients of air $\mu_{\text {air }}$, particles $\mu_{\text {particles }}$ and suspending fluids $\mu_{\text {fluid }}$ are well separated and sorted according to:

$$
\mu_{\text {air }}<\mu_{\text {particles }}<\mu_{\text {fluid }}
$$

To get rid of false detections, we build an histogram of the values of absorption of the detected extrema (Fig. 6). In practice, we do not use the value of the extrema, but the averaged value over a close neighbourhood around the extrema position, of 


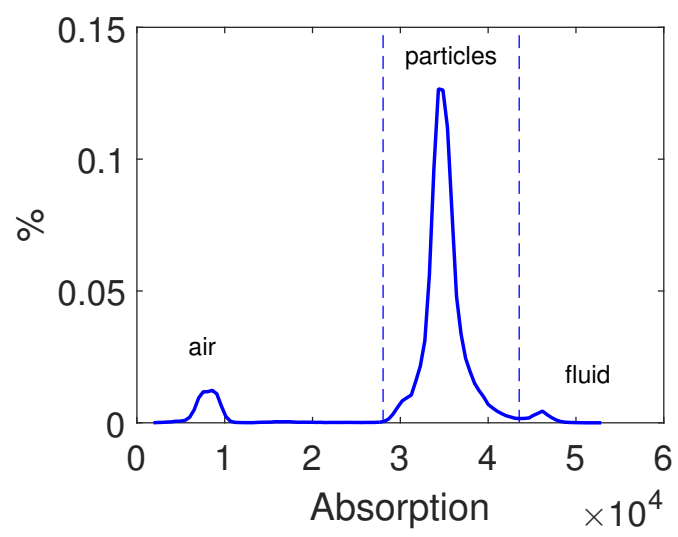

FIG. 6. Histogram (in fraction) of absorption values (without unit measurement) averaged over a close neighbourhood of size $r_{n e i g h}$ around the detected extrema, for the visco-plastic suspension at a solid fraction $\phi \simeq 35 \%$, before selection of extrema identified as real particles.

size $r_{\text {neigh }}$ taken equal to $60 \%$ of the mean diameter of particles (Tab. II), to avoid considering small dust (solid of tiny size). Also, this allows for a better separation in the histogram. On the histogram, we clearly distinguish the absorption values corresponding to particles, fluid and air. We kept only voxels of averaged absorption values between $\mu_{\min }$ and $\mu_{\max }$. In Figure 6, the width of the histogram results from the macroscopic inhomogeneities of absorption in space (due to a phenomenon called beam hardening) and likely bears the signature of particle distribution in space. False detections corresponding to local extrema in the plates have the same absorption value as particles, so a meticulous inspection of slices close to the plates and a manual exclusion may be needed if necessary.

- We compute distances between possible particle center positions to get rid of multiple detections. If two possible centers are closer than a threshold distance $r_{m i n}$, we keep only the one of minimal absorption. In the case of perfectly monodisperse particles and non-noisy images of infinite spatial resolution, $r_{\text {min }}$ could be taken as $100 \%$ of the diameter of particles. Since particles are slightly polydisperse and at this step the accuracy of particle centers is only of \pm 1 voxel, we choose $r_{\min }$ equal to $50 \%$ of the mean diameter of particles (Tab. II). Figure 7a shows the apparent volume fraction $\phi_{\infty}$ far from reference particles, computed from the number of neighbours detected by unit volume, for different values of the threshold distance $r_{\text {min }}$. The 

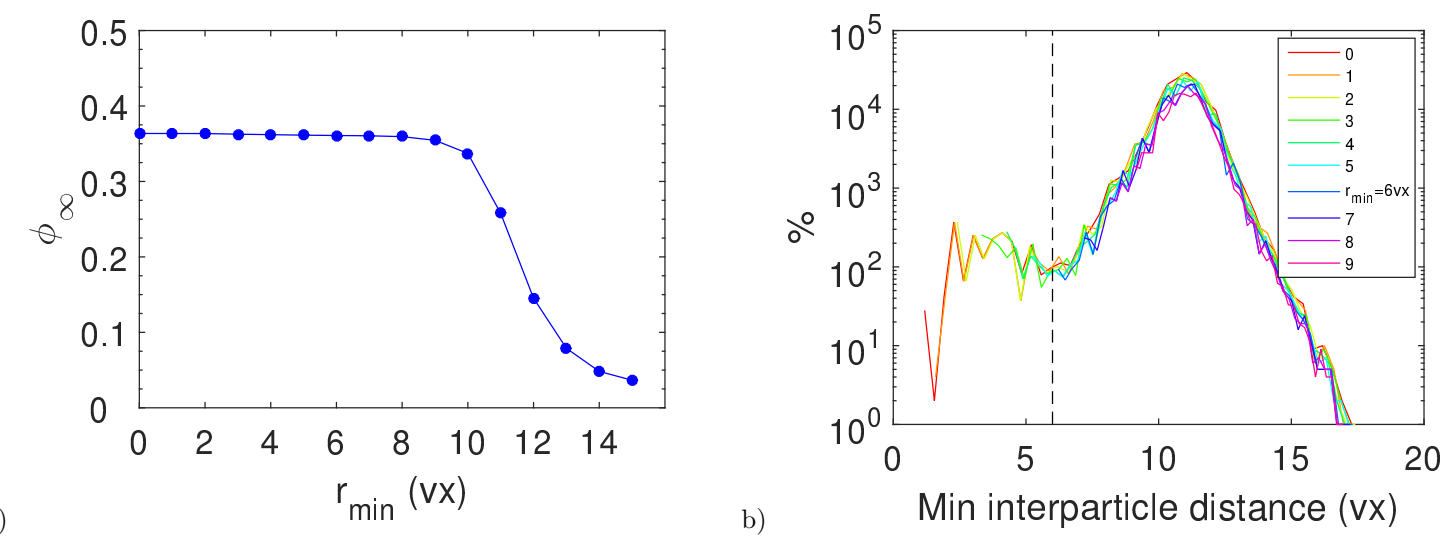

FIG. 7. a) Volume fraction $\phi_{\infty}\left(r_{\text {min }}\right)$ far from reference particles, computed from the number of neighbours detected by unit volume, for different values of the threshold distance $r_{\min }$ (minimal distance between two centers). b) Distribution of the interparticle distance for the identified first neighbours for all $r_{\min } \leq 9$. The visco-plastic suspension $(\mathrm{a}, \mathrm{b})$ has a solid fraction equal to $\phi \simeq 36 \%$. 328 influence of their values on our results. Finally, we do not observe any forgotten particle 329 when we systematically check some samples of 3D images. 


\section{B. Sub-voxel identification of particle centers}

331 At this stage, the selected centers corresponding to exact voxel positions are identified 332 as particles. We then refine their positions at a sub-voxel resolution by using symmetry 333 properties of the absorption signal over a particle around its center (Fig. 4).

334 First, the filtered image $I_{f}$ is linearly interpolated at the chosen sub-voxel spatial resolu335 tion to give $I_{v x}$ (the resolution we want for the particles centers). The (tri)linear interpolation 336 lays on the following principle: the value of the voxel to interpolate (at the chosen position) 337 is taken equal to the average of the values of the (original) crossed voxels, weighted by the 338 relative partial crossed volumes.

339 Second, an algorithm searches the position $\left(x_{0}, y_{0}, z_{0}\right)$ in a range $\pm\left(\delta x_{0}, \delta y_{0}, \delta z_{0}\right)$, mini340 mizing $\Delta I$, built to quantify the asymmetry of the signal around this center, defined as:

$$
\Delta I^{2}=\sum_{\Delta r \leq r_{s y m}}\left(I_{v x}\left(x_{0}+\Delta x, y_{0}+\Delta y, z_{0}+\Delta z\right)-I_{v x}\left(x_{0}-\Delta x, y_{0}-\Delta y, z_{0}-\Delta z\right)\right)^{2}
$$

341 for values of $\Delta r=\sqrt{\Delta x^{2}+\Delta y^{2}+\Delta z^{2}}$ smaller than a threshold $r_{\text {sym }}$, taken as $60 \%$ of the 342 mean diameter of particles (Tab. II).

343 A compromise between precision and computer time leads us to refine successively the 344 centers positions at 1 voxel first, then $1 / 2$ voxel, until $(1 / 2)^{n}$ voxel, with $n$, the integer such 345 that the chosen spatial resolution is reached.

346 Figure 8a shows the variations of $\Delta I$ with the displacement $\Delta x$ of the center in the $x$ 347 direction in the range \pm 2 voxels, for different couple values $(\Delta y, \Delta z)$ of the displacement of 348 the center in the $y$ - and $z$-directions: there is indeed a minimum $\Delta I_{\min }$ allowing to identify 349 the center. The same holds for $\Delta y$ and $\Delta z$ in the $y$-and $z$-directions. Figure $8 \mathrm{~b}$ shows the 350 decrease of the minimum $\Delta I_{\min }$ with $n$, the step of sub-voxel identification, what confirms 351 that the minimization and the sub-voxel refinement have still a physical sense till $n=5$, 352 allowing to reach a resolution on the particles center position of $(1 / 2)^{5}$ voxel $=0.375 \mu \mathrm{m}$ ${ }_{353} \simeq \mathrm{d} / 100$. Note that $n=-1$ (Fig. $8 \mathrm{~b}$ ) corresponds to the case of no interpolation of the 354 images $I_{f}$, so that $\Delta I_{\text {min }}(n=-1)$ quantifies the asymmetry of the signal around the minimal 355 absorption voxel. For the results presented in this paper, a spatial resolution of $6 \mu \mathrm{m} \simeq \mathrm{d} / 20$, 356 i.e. $n=1$ is enough.

357 Figure 9 shows the original (non interpolated, i.e. at 1 voxel resolution, $n=-1$ ) absorp358 tion signal $I_{f}$ (without unit measurement) over one particle as a function of distance from 

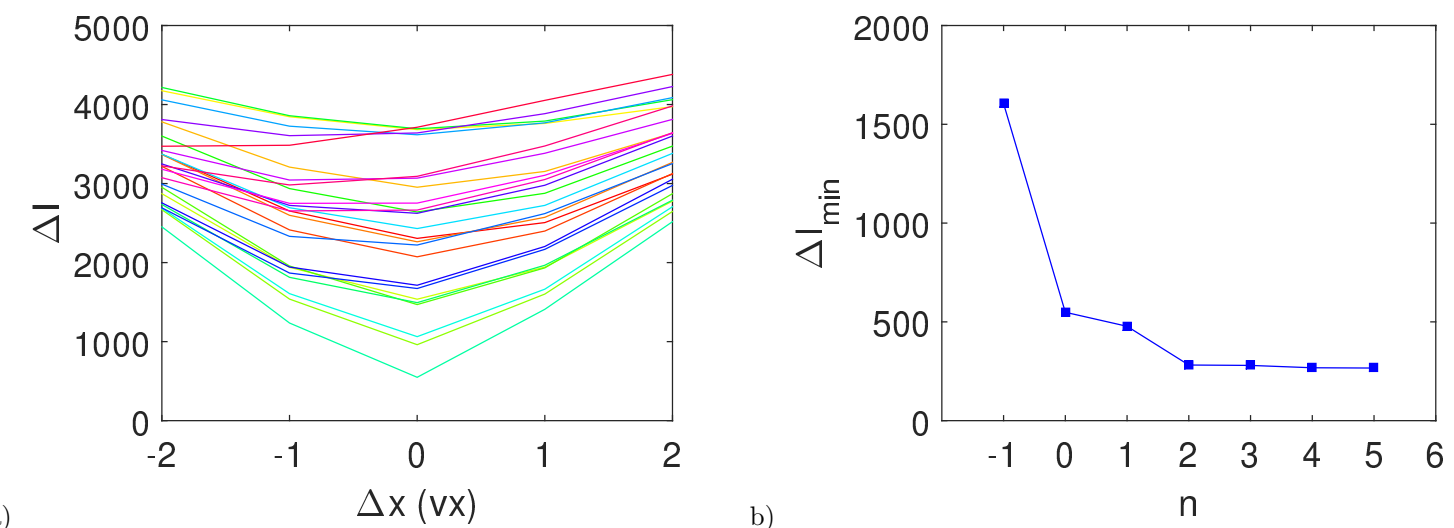

FIG. 8. a) Variations of $\Delta I$ (Eq. 7 ) with the displacement $\Delta x$ of the center in the $x$-direction for the first step of sub-voxel identification $n=0$. Each line corresponds to different couple values of the displacement of the center $(\Delta y, \Delta z)$ in the $y$ - and $z$-directions. b) Variations of $\Delta I_{\min }$ with the number of steps of sub-voxel identification $n . \Delta I(n=-1)$ quantifies the asymmetry of the signal around the minimal absorption voxel, i.e. without any interpolation of the image $I_{f}$. Note that here $\Delta I$ was normalized by the number of voxels for which it has been computed to allow for a comparison at different sub-voxel resolutions

359 the center, for the initial center (determined from the local extrema) (Fig. 9a) and for the 360 centers refined successively with $n=0,1$, and 2 in Figure 9b, c and d respectively. We see 361 that for $\Delta r \leq r_{\text {sym }}$ the scattering of data (or asymmetry of the signal around its center) 362 decreases at each step of sub-voxel identification, that is quantified by $\Delta I$. Even at 1 voxel 363 resolution (for $n=0$ ), we already note that the center of symmetry (which is used to plot ${ }_{364}$ Figure 9b) is not always the minimal voxel of absorption (which is used to plot Figure 9a).

\section{Pair distribution functions}

From particle positions within the parallel plates geometry, we are able to study how 367 particles are spatially distributed in the suspending fluid. In addition to information on the 368 structure of the suspension at the macroscopic scale from global positions of particles within 369 the geometry, we do get information on the microstructure of the suspension at the particle 370 scale from relative positions of particles (relative to each other) through the computation of 371 pair distribution functions [7].

The pair distribution function $g(\vec{r})$ is the probability of finding a particle pair separated 

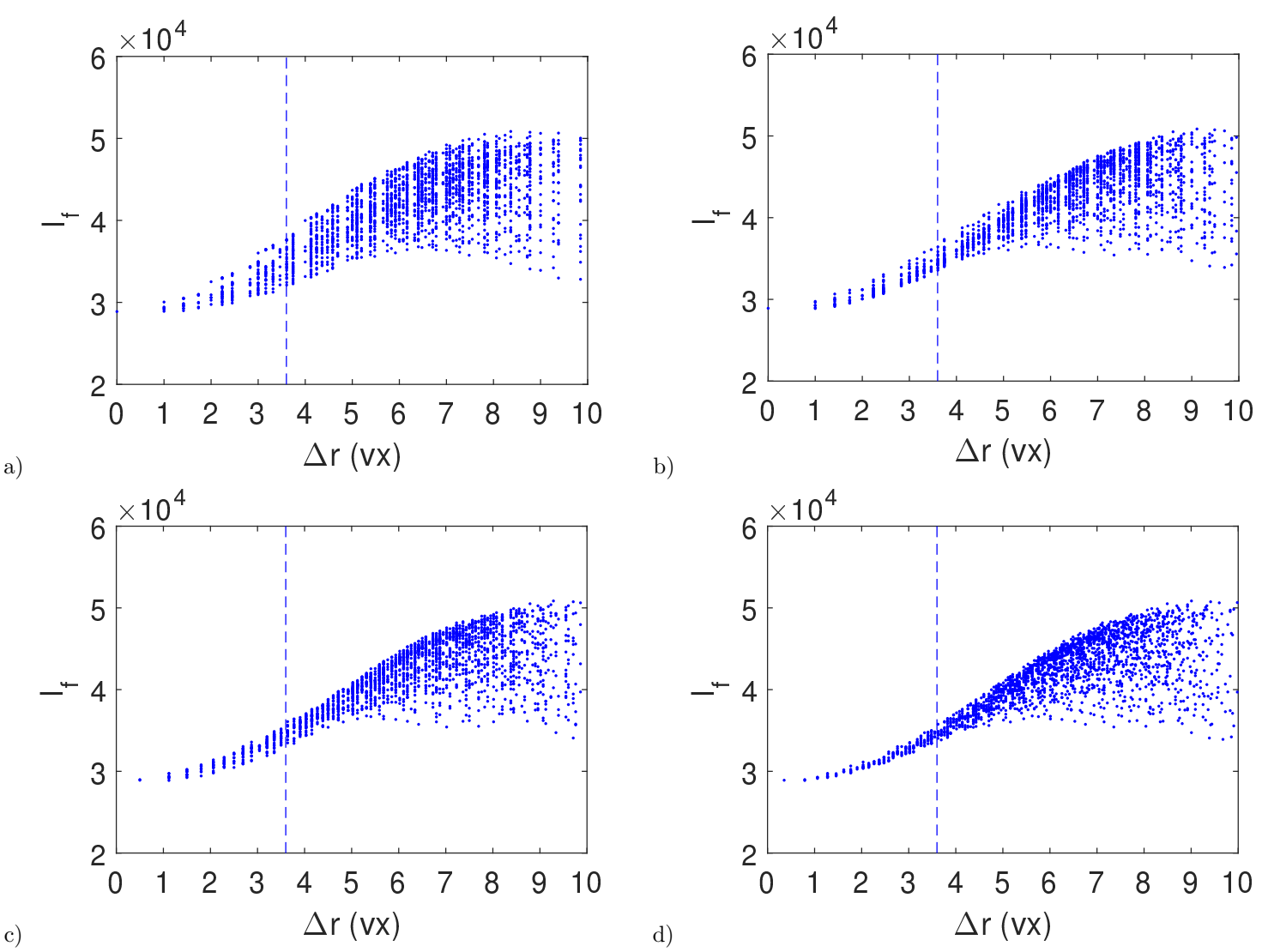

b)

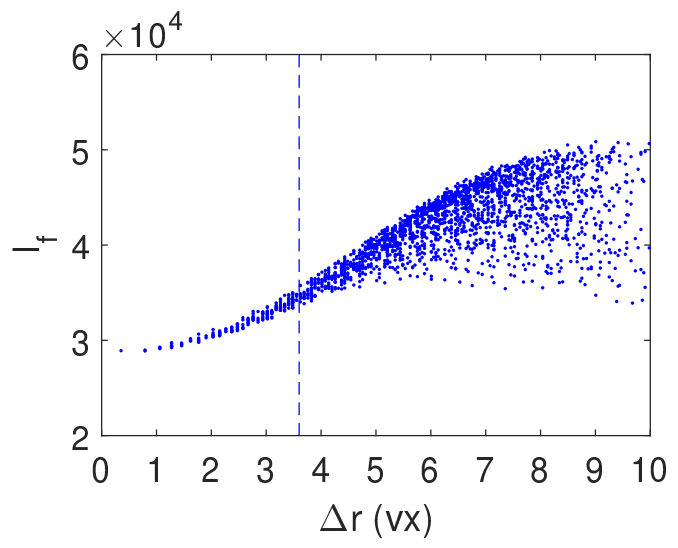

FIG. 9. Absorption levels (without unit measurement) of voxels over one particle and its close neighbourhood in the visco-plastic suspension at a solid fraction $\phi \simeq 40 \%$ as a function of the distance $\Delta r$ to its center, through the different steps $(n=-1,0,1,2$ in a, b, c, d) of sub-voxel identification based on symmetrisation

373 by the vector $\vec{r}$ normalized by the mean particle density, so that the asymptotic value of $g$ 374 for large values of $\|\vec{r}\|$ is:

$$
g(|| \vec{r} \| \rightarrow \infty)=1,
$$

375 in the absence of macroscopic structure or long-range correlations. Equivalently, $g(\vec{r})$ is a 376 normalized probability of finding a (test) particle located at $\vec{r}_{0}+\vec{r}$ with another (reference) 377 particle located at $\vec{r}_{0}$. This last definition suggests naturally how to compute $g(\vec{r}): N_{r e f}$ 378 particles are subsequently chosen to be the reference particles; we identify the $N^{\text {pair }}$ test 379 particles separated by $\vec{r}$ from each reference particle, corresponding to the number of pairs 380 characterized by $\vec{r}$ in the region of interest.

381 In the following, $\overrightarrow{r_{0}}=\left(r_{0}, \theta_{0}, z_{0}\right)$ and $\overrightarrow{r_{0}}+\vec{r}=(r, \theta, z)$ are the cylindrical coordinates 382 (attached to the axis of the rheometer circular plates) of a reference and of a test particle 


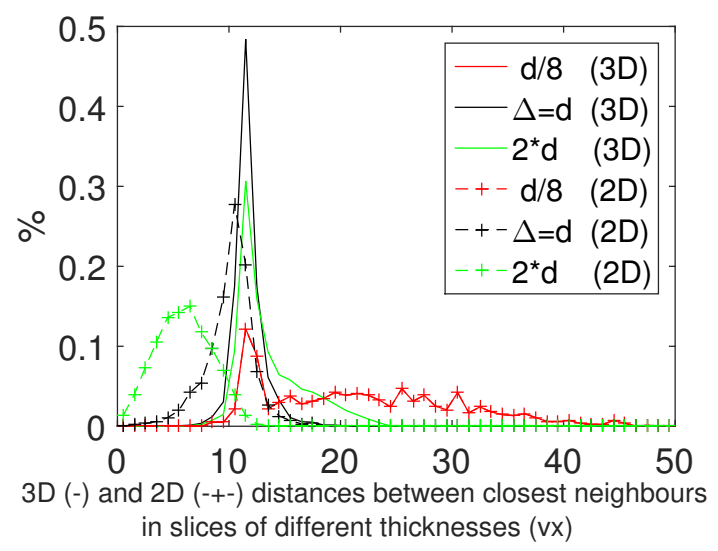

FIG. 10. Histogram (in fraction) of the interparticle 3D (solid lines) and 2D (dashed lines with crosses) distances for the first neighbours identified in slices of different thicknesses $\Delta=d / 8, d$ and $2 * d$, with $d$, the particles' diameter. The visco-plastic suspension has a solid fraction equal to $\phi \simeq 38 \%$.

383 located in $M_{0}$ and $M$.

384 To avoid any finite size or geometry effect on $g(\vec{r})$, that would introduce some bias 385 for example underestimating the statistics in the direction of a boundary - test particles 386 shall be selected around their reference particle within an isotropic region (e.g. that does 387 not cross any boundary). To be able to study the microstructure even close to boundaries 388 (solid plates or free interfaces), we use adaptative spheres of variable radius, equal to the 389 minimal distance between the reference particle and the closest boundary, as the area where 390 searching test particles around a reference particle. In this case, we have to update the 391 number of reference particles $N_{\text {ref }}$ that is no more constant for all values of $\vec{r}$, especially 392 for large values of $\|\vec{r}\|$. This choice is an alternative to exclusion of regions of investigation 393 as in [14] and to a geometry-dependent correction introduced in the computation of $g(\vec{r})$ as 394 in $[11]$.

395 The 3D pair distribution function $g(\vec{r})$ is a function of 3 scalar variables of size $\sim N^{3}$ in 396 the discretized space, with $N$, the number of voxels in a given direction of space. However, 397 for visualization, we generally plot the values of $g(\vec{r})$ taken in three orthogonal slices (data 398 of size $\sim N^{2}$ ). As relevant slices, we choose the cylindrical 'planes' attached to the axis of 399 the rheometer circular plates, as naturally suggested by the cylindrical symmetry of the set$400 \mathrm{up}$ and the flow. So, to reduce computation time, we generally compute only three 2D pair 
${ }_{401}$ distribution functions: $g_{r}$ in the orthoradial 'plane' $(\theta z)$ normal to $r$ (being a toroidal ring or 402 a cylinder), $g_{\theta}$ in the radial plane $(r z)$ normal to $\theta$ and $g_{z}$ in the horizontal plane $(\theta r)$ normal 403 to $z$. So, the region where searching for test particles can be reduced to the intersection of 404 a sphere and the elementary cylindrical 'plane' of interest attached to the reference particle. 405 In practice, defining the elementary planes as strictly 2D slices of cylindrical coordinates ${ }_{406} r=r_{0}, \theta=\theta_{0}$ or $z=z_{0}$ for a reference particle in $M_{0}\left(r_{0}, \theta_{0}, z_{0}\right)$ does not allow to sample ${ }_{407}$ enough test particles and get enough statistics, so we affect elementary thicknesses to the 408 cylindrical 'planes'. Whereas the definition of the thicknesses $\Delta z$ of horizontal planes $(\theta r)$ 409 and $\Delta r$ of orthoradial 'planes' $(\theta z)$ is obvious, the thickness of radial planes $(r z)$ offers ${ }_{410}$ several choices (see Appendix A). We choose the definition of:

$$
\Delta h=r \sin \left(\theta-\theta_{0}\right),
$$

${ }_{411}$ corresponding to a constant euclidean thickness (or minimal distance of $M$ from the ra${ }_{412}$ dial direction $O M_{0}$ ), because it prevents a non symmetric pattern on the pair distribution ${ }_{413}$ function $g_{\theta}$ (see Appendix A). We checked the influence of the value $\Delta$ of the thicknesses ${ }_{414}$ of the planes $\Delta=\Delta z=\Delta r=\Delta h$ on our results. Figure 10 compares two distributions: 415 the 3D distances (real distance) and the 2D distances (projected in the slice) between first ${ }_{416}$ neighbours of particles in an elementary cylindrical plane for different thicknesses $\Delta$ be${ }_{417}$ tween $d / 8$ and $2 * d$. The 3D distances are always equal or larger than $d$ with a maximum ${ }_{418}$ probability for $d$. But the 2D distances are not, because they do not represent well real 3D 419 distances in slices of thicknesses larger than $d$, as demonstrated by the distributions that do ${ }_{420}$ not super-impose for $\Delta>d$. This leads us to choose for the thickness of elementary cylin${ }_{421}$ drical 'planes' $\Delta=d$, with $d$ the particle diameter, as a compromise between accuracy and ${ }_{422}$ statistics. Moreover we checked that this choice allows to preserve the quality and quantity ${ }_{423}$ of the pdfs.

${ }_{424}$ Different choices for the characterization of the pair vector $\vec{r} \equiv \overrightarrow{M_{0} M}$ (relative coordinates 425 between test and reference particles located in $M$ and $M_{0}$ ) are possible (see Appendix A). ${ }_{426}$ Euclidean coordinates can in principle be chosen but we believe that non euclidean coor${ }_{427}$ dinates, curvilinear projected along the cylindrical 'planes' aligned with the circular flow ${ }_{428}$ streamlines, are more relevant to characterize the microstructure induced by a simple shear ${ }_{429}$ flow. We choose for coordinates of the pair vector $\vec{r}=(\rho, \ell, \xi)$ :

$$
\rho=r-r_{0}, \ell=r\left(\theta-\theta_{0}\right), \xi=z-z_{0},
$$




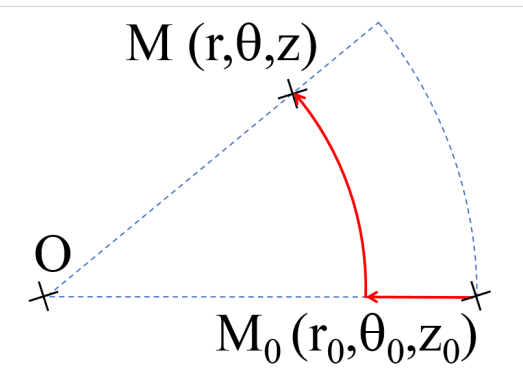

FIG. 11. Two particles $M_{0}\left(r_{0}, \theta_{0}, z_{0}\right)$ and $M(r, \theta, z)$ in the global cylindrical framework and definition of the pair vector $\vec{r} \equiv \overrightarrow{M_{0} M}$ characterizing their separation in curvilinear coordinates (along circular flow lines $): \vec{r}=(\rho, \ell, \xi)=\left(r-r_{0}, r\left(\theta-\theta_{0}\right), z-z_{0}\right)$

430 as shown in Figure 11. This choice does not induce any bias in the symmetry of the pair 431 distribution functions $g_{r}, g_{\theta}$ or $g_{z}$ (see Appendix A). Note that in the following, when showing 432 a $2 \mathrm{D}$ pdf $g\left(g_{r}, g_{\theta}\right.$ and $\left.g_{z}\right)$, we will use alternatively cartesian coordinates (couples among $\rho$, ${ }_{433} \ell$ or $\left.\xi\right)$ or polar coordinates $\left(\rho_{2 d}, \phi_{2 d}\right)$ for a pair of particles in the plane of interest. In the ${ }_{434}(\theta z),(r z)$ and $(\theta r)$ planes, cartesian and polar coordinates are respectively related through ${ }_{435}\left(\rho_{2 d} \cos \phi_{2 d}, \rho_{2 d} \sin \phi_{2 d}\right)=(\ell, \xi),(\rho, \xi)$ and $(\rho, \ell)$.

${ }_{437}$ Altogether, with the mean particle density (or number of particles per unit volume):

$$
n_{0 v}=N / V=\phi /\left(\pi d^{3} / 6\right)
$$

438 with the total number of particles $N$, the total volume of the suspension $V$, the particle 439 concentration (or solid fraction) $\phi$, the particle diameter $d$, the formula for the $2 \mathrm{D}$ pair ${ }_{440}$ distribution functions $g_{r}, g_{\theta}$ and $g_{z}$ in the $(\theta z),(r z)$ and $(\theta r)$ planes respectively (for spatial ${ }_{441}$ resolutions $\delta \rho$ and $\left.\delta \ell, \delta \xi\right)$ are:

$$
\begin{aligned}
& { }_{442} \quad g_{r}(\ell, \xi)=P_{r}(\ell, \xi) / n_{0 v}, \text { with } P_{r}(\ell, \xi) \delta V_{r}=N_{r}^{\text {pair }}(\ell \pm \delta \ell / 2, \xi \pm \delta \xi / 2) / N_{r e f}, \\
& { }_{443} \quad g_{\theta}(\rho, \xi)=P_{\theta}(\rho, \xi) / n_{0 v}, \text { with } P_{\theta}(\rho, \xi) \delta V_{\theta}=N_{\theta}^{p a i r}(\rho \pm \delta \rho / 2, \xi \pm \delta \xi / 2) / N_{r e f}, \\
& { }_{444} \quad \bullet g_{z}(\rho, \ell)=P_{z}(\rho, \ell) / n_{0 v}, \text { with } P_{z}(\rho, \ell) \delta V_{z}=N_{z}^{\text {pair }}(\rho \pm \delta \rho / 2, \ell \pm \delta \ell / 2) / N_{r e f},
\end{aligned}
$$

${ }_{445}$ with $N_{\text {ref }}$, the number of reference particles, and $(\rho, \ell, \xi)$, the coordinates of a pair vector ${ }_{446}$ defined in Eq. (10). While $g$ refers to a pair distribution function, $P$ refers to a probability ${ }_{447}$ of finding a pair of particles and $N^{\text {pair }}$ to a number of pairs of particles. More precisely, ${ }_{448} N_{z}^{\text {pair }}(\rho \pm \delta \rho / 2, \ell \pm \delta \ell / 2)$ is the number of pairs of coordinates $(\rho \pm \delta \rho / 2, \ell \pm \delta \ell / 2)$ in cylindrical 
${ }_{449}$ horizontal planes $(\theta r)$ of thickness $\Delta z$ or equivalently the number of test particles in the 450 same horizontal plane than the reference particles $\left(\right.$ at $z_{0}$ ), i.e. particles at $z_{0} \pm \Delta z / 2$; the ${ }_{451}$ same holds for $N_{r}^{\text {pair }}(\ell \pm \delta \ell / 2, \xi \pm \delta \xi / 2)$ and $N_{\theta}^{\text {pair }}(\rho \pm \delta \rho / 2, \xi \pm \delta \xi / 2)$ with the thickness ${ }_{452} \Delta r$ and $\Delta h$ of orthoradial plane $(\theta z)$ and radial plane $(r z)$ respectively. The elementary 453 sampling volumes (defining the spatial resolution for $g$ in 2D) are equal to:

$$
\delta V_{r}=\Delta r \delta \ell \delta \xi, \delta V_{\theta}=\delta \rho \Delta h \delta \xi, \text { and } \delta V_{z}=\delta \rho \delta \ell \Delta z
$$

${ }_{454}$ Here, the thickness of the 'planes' are chosen as $\Delta z=\Delta r=\Delta h=d$ and usually the spatial 455 resolution for pair distribution functions $g$ is chosen as $\delta \rho=\delta \ell=\delta \xi=1 / 2$ voxel. Note ${ }_{456}$ that these spatial averages may lead to some underestimate of peak values of $g$ whether 457 there are large variations of $g$, that may lead to some discrepancies between theoretical and ${ }_{458}$ experimental values. Finally, in three dimensions, the expression for the pdf $g$ is:

$$
g(\vec{r} \pm \delta \vec{r})=N^{\text {pair }}(\vec{r} \pm \delta \vec{r}) / N_{\infty}^{\text {pair }} \text { with } N_{\infty}^{\text {pair }}=N_{r e f} n_{0 v} \delta V
$$

${ }_{459} N_{\infty}^{\text {pair }}$ being the number of pairs separated by a vector $\vec{r}$ such that $\|\vec{r}\| \rightarrow \infty$ in the sampling 460 volume $\delta V$ in the space of pair vectors delimited by $\vec{r} \pm \delta \vec{r}$.

461 In the following, the analysis of the microstructure will be generally realized, unless spec${ }_{462}$ ified otherwise, far enough from the free interface and from the solid plates, to ensure not to ${ }_{463}$ mix possible structurations near boundaries to bulk microstructure. In order to characterize ${ }_{464}$ the microstructure for a roughly homogeneous simple shear, it has to be computed at a fixed ${ }_{465}$ radial position $R_{0}$ in the parallel plates geometry. In practice, to get a good precision of ${ }_{466}$ the pdf, a toroidal region of sufficient thickness $\Delta R$ has to be analysed. As an example (see ${ }_{467}$ Appendix B for details), if we want a precision $\Delta g=10^{-2}$ on $g(\vec{r})$ and a spatial resolution ${ }_{468}$ for the $2 \mathrm{D} \operatorname{pdf} \delta V=\Delta \delta^{2}$ with $\Delta=\mathrm{d}=140 \mu \mathrm{m}=12 \mathrm{vx}$ and $\delta=1 \mathrm{vx}=12 \mu \mathrm{m}$, for a solid ${ }_{469}$ fraction $\phi=35 \%\left(n_{0 v}=244 \mathrm{~mm}^{-3}\right)$, we need at least $2.10^{4}$ reference particles in the region 470 of interest. This leads us to choose $R_{0}=0.72 D / 2 \pm 0.12 D / 2$, unless specified.

${ }_{471}$ In the following, we now illustrate the potential of our technique by analyzing the mi472 crostructure of our suspensions near the horizontal solid plates, near the orthoradial free 473 interface, and in the bulk separately. 

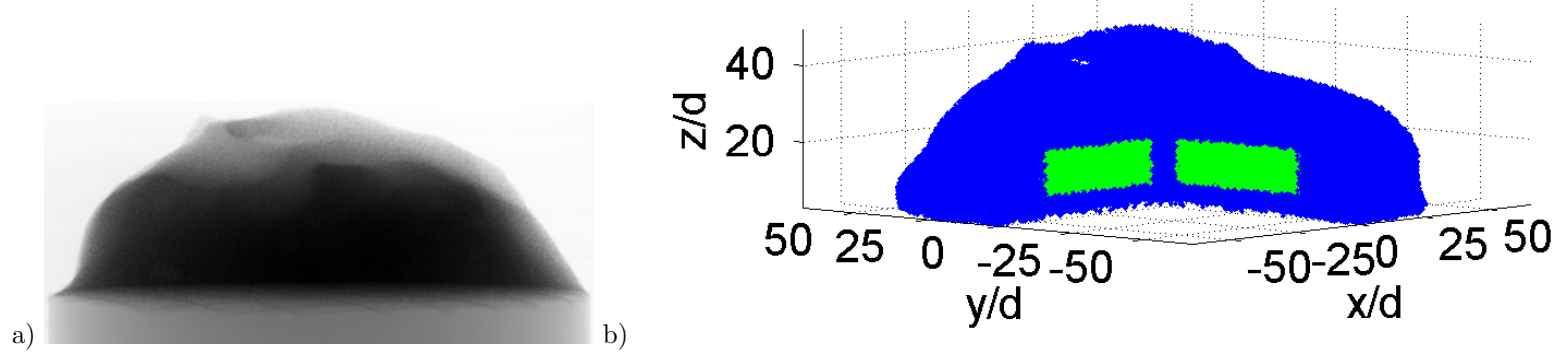

FIG. 12. a) X-ray radiograph of a non sheared drop of a visco-plastic suspension at a solid fraction $\phi \simeq 35 \%$. b) 3D plot of centers of all detected particle (in blue) and reference particles (in green) for the characterization of the microstructure

\section{${ }_{474}$ IV. MICROSTRUCTURE}

\section{A. Bulk microstructure of a non sheared visco-plastic suspension}

476 First, we characterize the initial microstructure of a visco-plastic suspension poured on 477 the bottom plate of the rheometer, but before any loading and shear history. A suspension 478 is prepared by simply mixing 'by hand' the particles and the fluid in a cup with a spatula, 479 with the goal of achieving a mixing close to chaotic mixing. With this procedure, we aim to 480 prepare a material that is homogeneous and isotropic. It is then degased to remove possible 481 air bubbles (in a vacuum or in a centrifuge). Upon pouring, the visco-plastic suspension 482 has the shape of an irregular drop due to its yield stress (Fig. 12). Here, we analyze the 483 microstructure in a toroidal region $\left(R_{0}=0.36 \mathrm{D} / 2 \pm 0.30 \mathrm{D} / 2\right.$ and $\left.Z_{0}=2.4 H \pm 1.2 H\right)$ inside ${ }_{484}$ the drop far from solid surfaces and free interfaces (Fig. 12b).

485 In its initial configuration, we observe that the suspension has at first order an isotropic 486 microstructure: the pair distribution function $g(\vec{r})$ does not depend much on the direction ${ }_{487} \vec{r} /\|\vec{r}\|$ (Fig. 13 and 19). Figure 13 shows $g_{r}(\ell, \xi), g_{\theta}(\rho, \xi)$ and $g_{z}(\rho, \ell)$ : they are roughly 488 the same in the three orthogonal 2D 'planes', with a circular symmetry in each 2D 'plane'. ${ }_{489} g(\vec{r})$ mostly depends only on the distance $\|\vec{r}\|$. Figure 14b shows the 1D scalar pdf $g(\|\vec{r}\|)$ : ${ }_{490} g(\|\vec{r}\|)=0$ for (and only for) distances $\|\vec{r}\| \lesssim d$; it has a maximal value for $\|\vec{r}\| \simeq d$ and a 491 second local maxima for $\|\vec{r}\| \simeq 2 d$; then $g$ tends to 1 for larger values of $\|\vec{r}\|$.

${ }_{492}$ Note that a closer inspection of the 2D pdfs (Fig. 13b) shows that they are not exactly 493 the same and not exactly isotropic: the averages of $g_{r}$ and $g_{\theta}$ for distances $\rho_{2 d}$ close to its ${ }_{494}$ maxima $\left(\rho_{2 d}=d \pm d / 6\right)$ as a function of $\phi_{2 d}$ (represented in a polar plot) are not perfectly 
a)

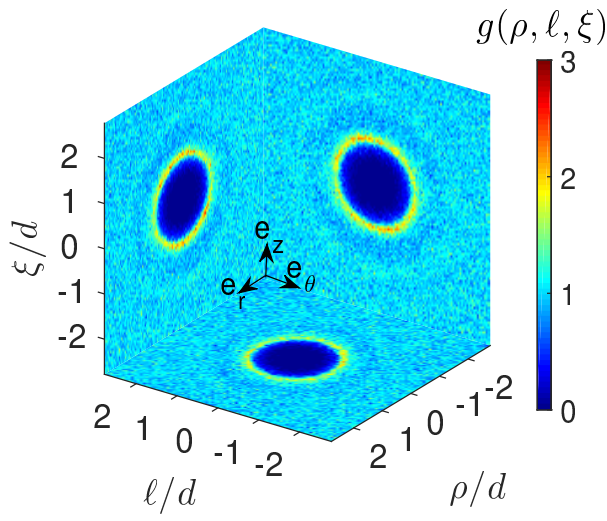

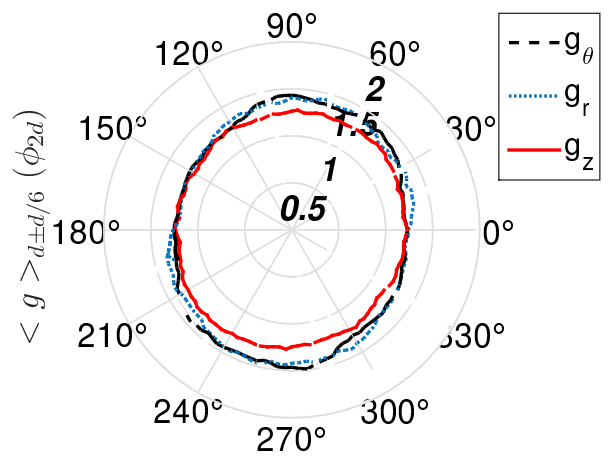

b)

FIG. 13. a) Three dimensional microstructure of a visco-plastic suspension in a non sheared configuration (drop) at a solid fraction $\phi \simeq 35 \%$ : 2D pair distribution functions $g_{r}(\ell, \xi), g_{\theta}(\rho, \xi)$ and $g_{z}(\rho, \ell)$. b) Polar plots of $\left\langle g\left(\phi_{2 d}\right)>_{\rho_{2 d}=d \pm d / 6}\right.$ in the three planes of interest $(\theta z),(r z)$ and $(\theta r)$
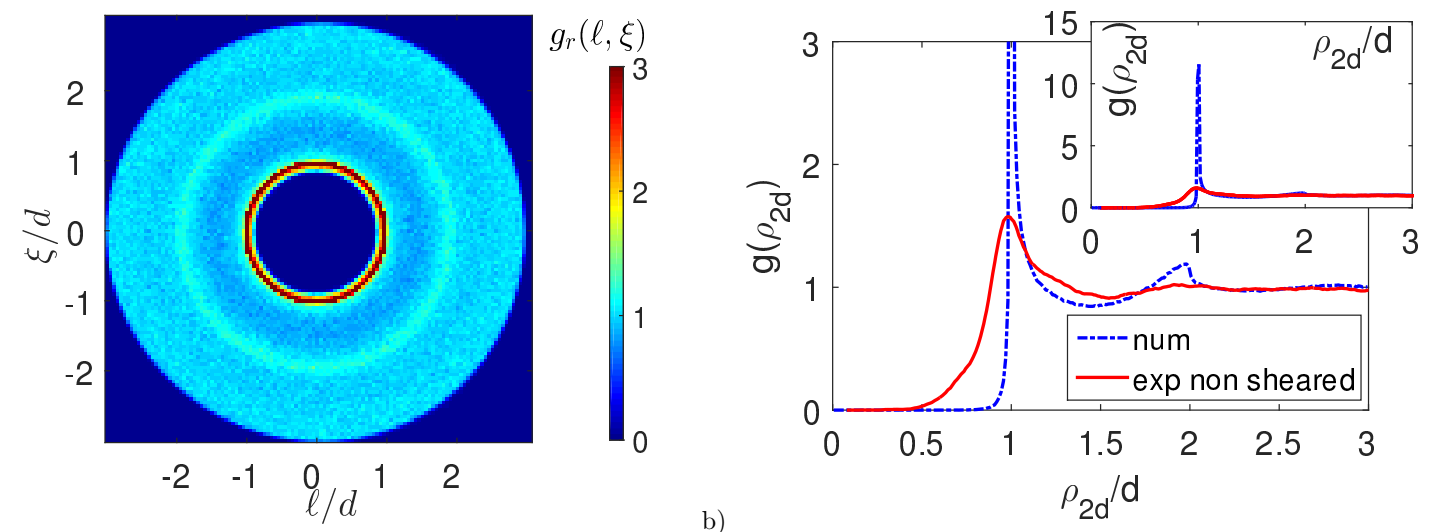

FIG. 14. Numerical microstructure from a simulation of finite-size particles following a naive rule of exclusion and redistribution for a solid fraction $\phi \simeq 36 \%$ : a) 2D pair distribution function $g(\ell, \xi)$ and b) 1D scalar pair distribution function $g\left(\rho_{2 d}\right)$ super-imposed with the experimental 1D pdf for a non sheared suspension

495 circular but bear a signature of a slight over-population of pairs of particles roughly aligned 496 with the gravity. This slight micro-structuration is not visible in the $(\theta r)$ plane (on $g_{z}$ ). ${ }_{497}$ This may be attributed to vertical flows of the suspension when it is poured on the plate. ${ }_{498}$ This almost isotropic experimental pdf is now compared to the numerical pdf obtained 499 when simulating a random distribution of finite-size particles, with a naive rule of exclusion 500 and redistribution (Fig. 14). The finite-size effect introduced here, arbitrarily governed 501 (i.e. without introducing physical forces), has to be seen as a minimal steric constraint. A 

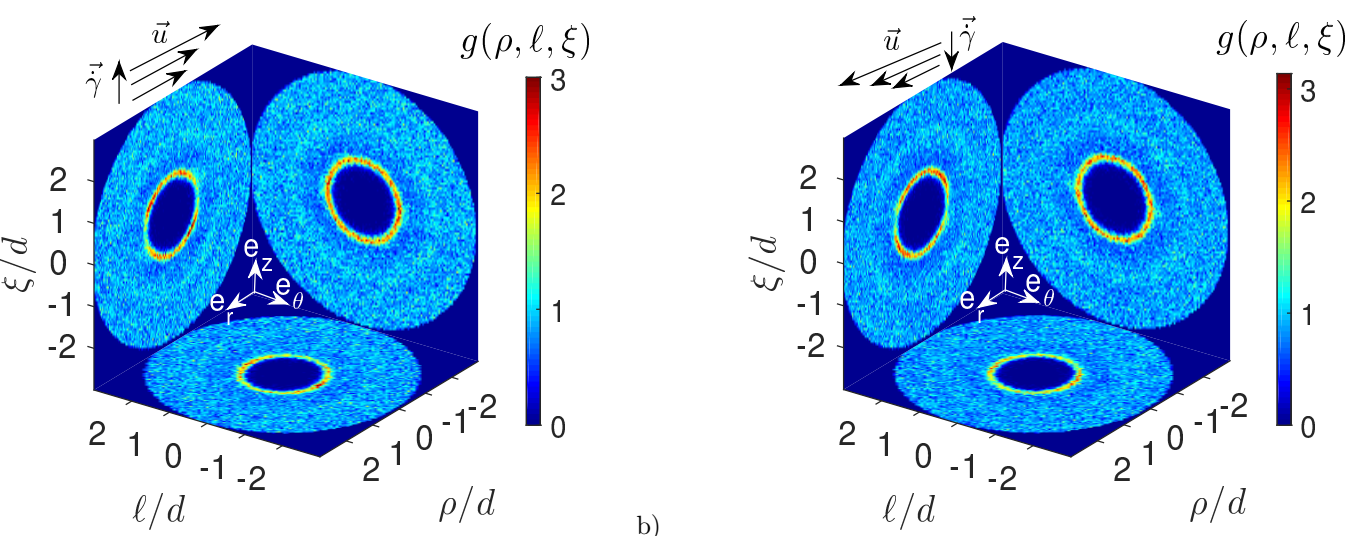

FIG. 15. Three dimensional microstructure of a visco-plastic suspension after loading and squeezing in a parallel plates geometry at a solid fraction $\phi \simeq 35 \%$ : $2 \mathrm{D}$ pair distribution functions $g_{r}(\ell, \xi), g_{\theta}(\rho, \xi)$ and $g_{z}(\rho, \ell)$ for $z<0$ (a) and for $z>0$ (b)

502 random distribution of points (representing particles of zero size) gives obviously a constant ${ }_{503} \mathrm{pdf}$ equal to 1 everywhere. Adding an effect of a finite size by taking away over-lapping ${ }_{504}$ particles (separated by a distance smaller than their diameter $d$ ) and separating them by 505 their diameter $d$ in the initial direction (and repeating this until no more particles over-lap $506[34]$ ) leads to a pdf dependent on $\|\vec{r}\|$ (Fig. 14) with the same features as the experimental ${ }_{507}$ pdf we measure in 3D in a non sheared visco-plastic suspension, in particular local minimum 508 and secondary maximum at the same positions. The main visible difference is the width 509 and the value of the first peak of $g$ maybe due to the difference of granulometry of the 510 particles, to the arbitrary rule of redistribution of over-lapping particles and to a specific 511 spatial distribution of particles in a non sheared suspension. Finally, the fluid does not seem 512 to have any significant impact on the microstructure during the mixing.

\section{B. Bulk microstructure of a visco-plastic suspension after a continuous squeeze}

514 When a suspension is loaded into a parallel plates geometry, it is first poured on the 515 bottom plate. The top plate is then translated downwards, thus squeezing the visco-plastic 516 suspension between the two plates. The flow induced by this squeeze flow is an inhomoge517 neous simple shear flow, which has been thoroughly described in the literature [30-32] and 518 is briefly discussed in part II D.

${ }_{519}$ The technique developed here for the 3D characterization of microstructure allows us 

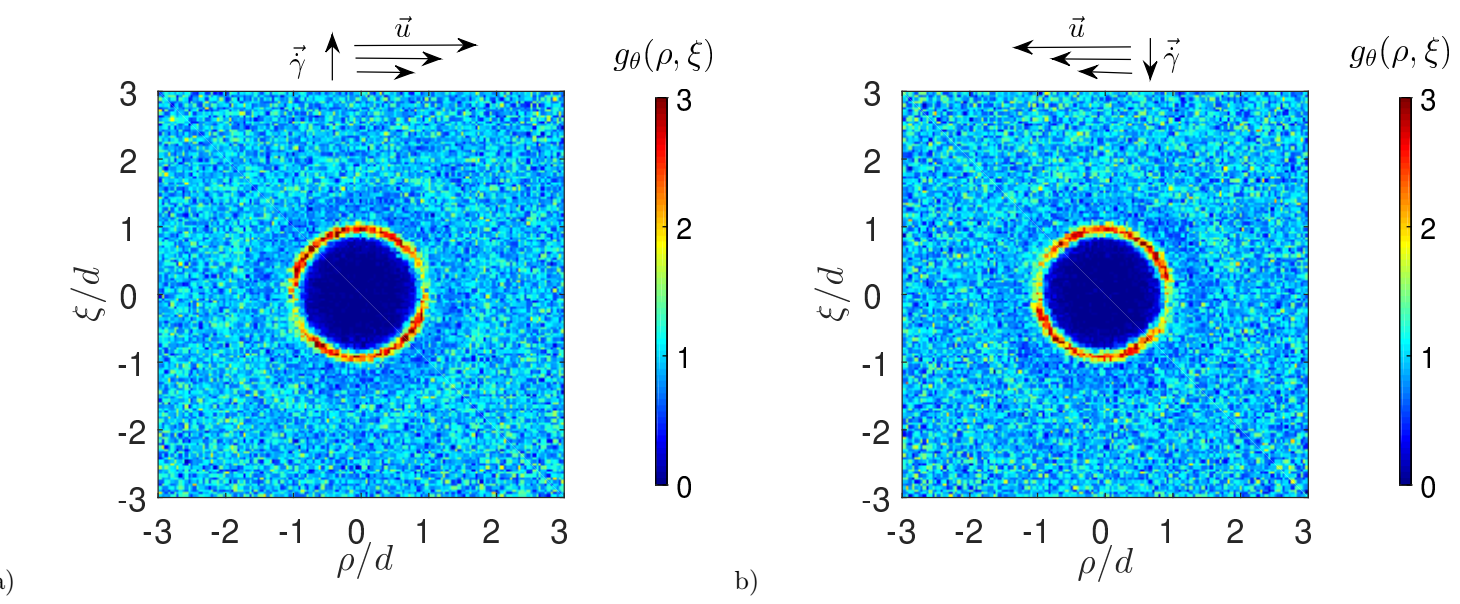

FIG. 16. Pair distribution function $g_{\phi}(\rho, \xi)$ after a squeeze flow in the shear plane for $z<0$ (a) and for $z>0(\mathrm{~b})$
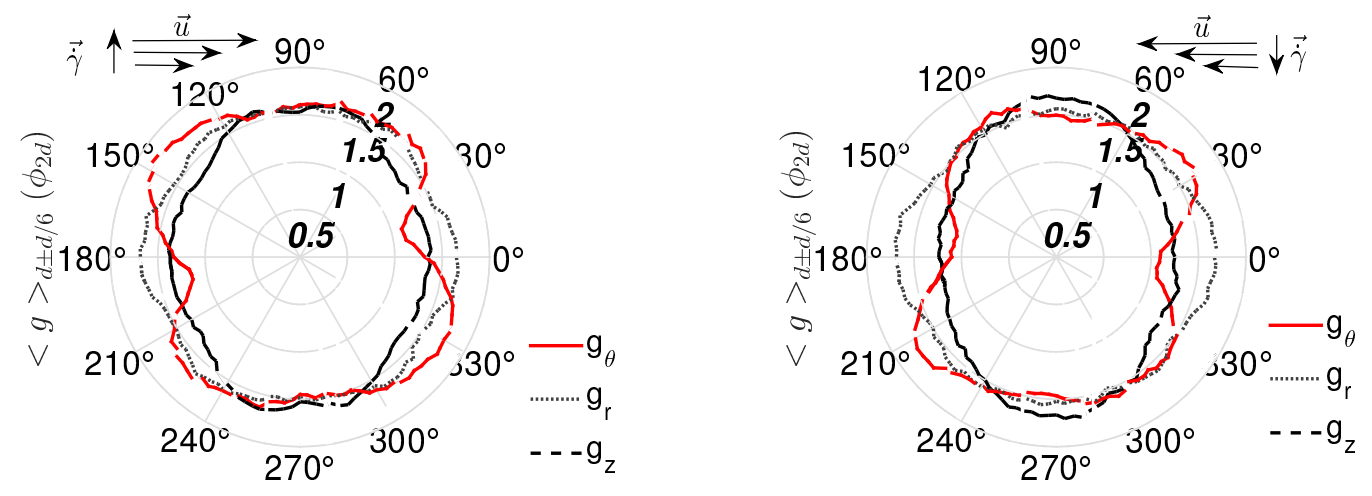

a)

b)

FIG. 17. Pair distribution functions $g_{r}, g_{\theta}$ and $g_{z}$ averaged for distances $\rho_{2 d}=d \pm d / 6$ plotted as a function of the angle $\phi_{2 d}$ in polar coordinates after a squeeze flow for $z<0$ (a) and for $z>0$ (b) for a visco-plastic suspension at a solid fraction $\phi \simeq 35 \%$

520 to investigate if and how the simple shear in the $(r z)$ plane induced by this squeeze flow 521 changes the microstructure of the suspension, initially nearly isotropic as seen above. The ${ }_{522}$ plane in the middle of the gap, defined by $z=0$, is an axis of symmetry of the flow. To take 523 into account the different sign of the shear rate (velocity gradient) in the upper and lower ${ }_{524}$ part of the suspension, we analyse here the microstructure separately in both semi-planes ${ }_{525} z<0$ and $z>0$.

526 In Figures 15 and 16, we observe that an anisotropic microstructure develops in the shear ${ }_{527}$ plane $(r z)$ (velocity-velocity gradient plane), while it remains approximately isotropic in the 
${ }_{528}$ two other cylindrical planes $(\theta z)$ and $(\theta r)$ where the suspension does not experience any 529 shear. Contrary to the nearly isotropic microstructure observed previously in the initial 530 configuration, the pdf $g(\vec{r})$ is no more simply a scalar function of $\|\vec{r}\|$ but depends both on 531 the distance $\|\vec{r}\|$ and the direction $\vec{r} /\|\vec{r}\|$. As in shear-induced structuration observed in the 532 literature in the case of the rotational shear of a Newtonian suspension [7, 9-14], we observe 533 here a depletion of particles in close contact $\left(\rho_{2 d} \simeq d\right)$ located in the extensional area close 534 to the direction of the velocity (near the angles $+15^{\circ}$ and $+195^{\circ}$ for $z<0$ and $+165^{\circ}$ and $535+345^{\circ}$ for $z>0$ ). Moreover, Figure 19 shows the 1D scalar plot of $g(\|\vec{r}\|)$ : its maxima is 536 larger and tightens (spreads less) in comparison with the case of the non sheared suspension. ${ }_{537}$ As previously seen, a closer inspection of the 2D pdfs (Fig. 17 and 19) by looking at 538 the averages of $g_{r}, g_{z}$ and $g_{\theta}$ for distances $\rho_{2 d}$ close to its maxima $\left(\rho_{2 d}=d \pm d / 6\right)$ as a 539 function of $\phi_{2 d}$ shows a secondary structuration in addition to the depletion of particles in 540 the $(r z)$ plane: $g_{r}$ and $g_{z}$ exhibit an 'hexagonal' shape, that may be the signature of a more 541 complex flow occurring in the parallel plates than a simple shear flow as approximated in ${ }_{542}$ the framework of lubrification when $D / H$ is large (the flow has also a velocity component 543 aligned with the direction of translation of the bottom plate, which might not be negligible). ${ }_{544}$ As a consequence, a suspension initially characterized by a nearly isotropic microstruc${ }_{545}$ ture in 3D, develops at first an anisotropic microstructure when loaded in the parallel plates 546 geometry, even before any imposed shear history. Such impact of loading on the microstruc547 ture of suspension should be observed in most rheometrical devices (cone-and-plate, Couette, ${ }_{548}$ Poiseuille, ...). If the characterization of an isotropic structure is needed, a possible rheo549 metrical tool is the vane in cup geometry, classically used to study gels: isotropy can be 550 achieved by chaotic mixing in the cup, and then the insertion of the vane into the cup should 551 not affect the material's structure.

${ }_{553}$ After the loading of the visco-plastic suspension in the parallel plates geometry, a simple 554 shear flow in the $(\theta z)$ plane is imposed thanks to the rotation of the top plate until a 555 stationary state is reached in terms of shear stress.

$556 \quad$ As already observed for a Newtonian suspension (numerically and experimentally) [7, 9557 14] and as reported in Ovarlez et al. [5] for a visco-plastic suspension, the microstructure 

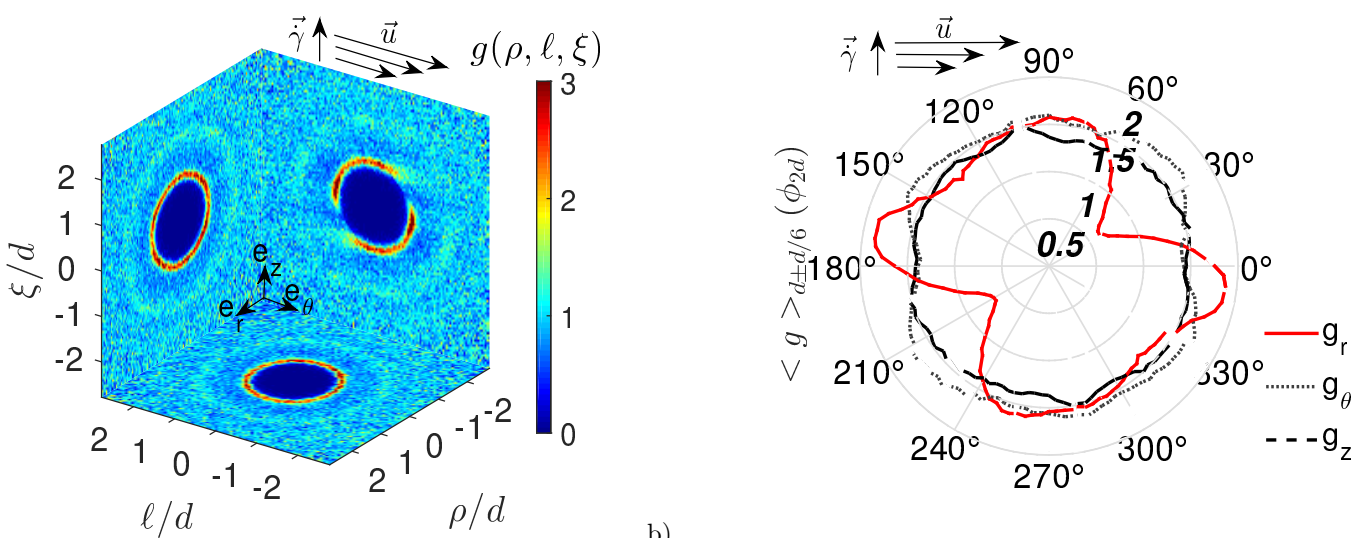

FIG. 18. a) Three dimensional microstructure: pair distribution functions $g_{r}(\ell, \xi), g_{\theta}(\rho, \xi), g_{z}(\rho, \ell)$ after a steady rotational shear at a shear rate $\dot{\gamma}=10^{-2} s^{-1}$ of a visco-plastic suspension at a solid fraction $\phi \simeq 35 \%$. b) Pair distribution function $g_{r}, g_{\theta}$ and $g_{z}$ averaged for $\rho_{2 d}=d \pm d / 6$ plotted as a function of the angle $\phi_{2 d}$ in polar coordinates.

558 becomes anisotropic in the $(\theta z)$ shear plane (velocity-velocity gradient plane), while it is ap559 proximately isotropic in the two other 'planes', as shown in Figure 18 and 19: this anisotropy 560 is referred to in the literature as a fore-aft asymmetry. In 3D, it means that the positions ${ }_{561}$ of particle pairs previously correlated in the $(r z)$ plane due to the squeeze flow, decorrelate ${ }_{562}$ thanks to the rotational shear and the particles reorganize themselves relatively to each 563 other, leading to some new correlations in the $(\theta z)$ plane.

564 These correlations between particles develop at the scale of particle pairs in close contact, 565 leading to a depletion of particles in the extensional area, in contrast with the compressional 566 area, as well as a primary over-population roughly aligned with the direction of the flow ${ }_{567}\left(+170^{\circ}\right)$. This anisotropy can be quantified thanks to the plots of $g\left(\phi_{2 d}\right)$ (the average of ${ }_{568} g\left(\rho_{2 d}, \phi_{2 d}\right)$ for $\left.\rho_{2 d}=d \pm d / 6\right)$ plotted in polar coordinates in Figure 18b: $g\left(\phi_{2 d}\right)$ has the 569 shape of a 'butterfly'.

570 More precisely, Figure 18 shows that for distances $\rho_{2 d}$ close to the value of the particles 571 diameter, $g_{r}$ has minimal values for the angles $+30^{\circ}$ and $+210^{\circ}$, corresponding to a decrease 572 of the number of pairs in the extensional stress domain; and it has maximal values for the 573 angles $+170^{\circ}$ and $+350^{\circ}$, corresponding to an increase of the number of particle pairs aligned 574 roughly with the flow. These two principal extrema can be referred to as an extensional575 depletion of particle pairs (for the minima of $g$ ) and a flow-alignment of particle pairs (for 576 the maxima of $g$ ). Whereas the extensional-depletion of particle pairs was already reported 

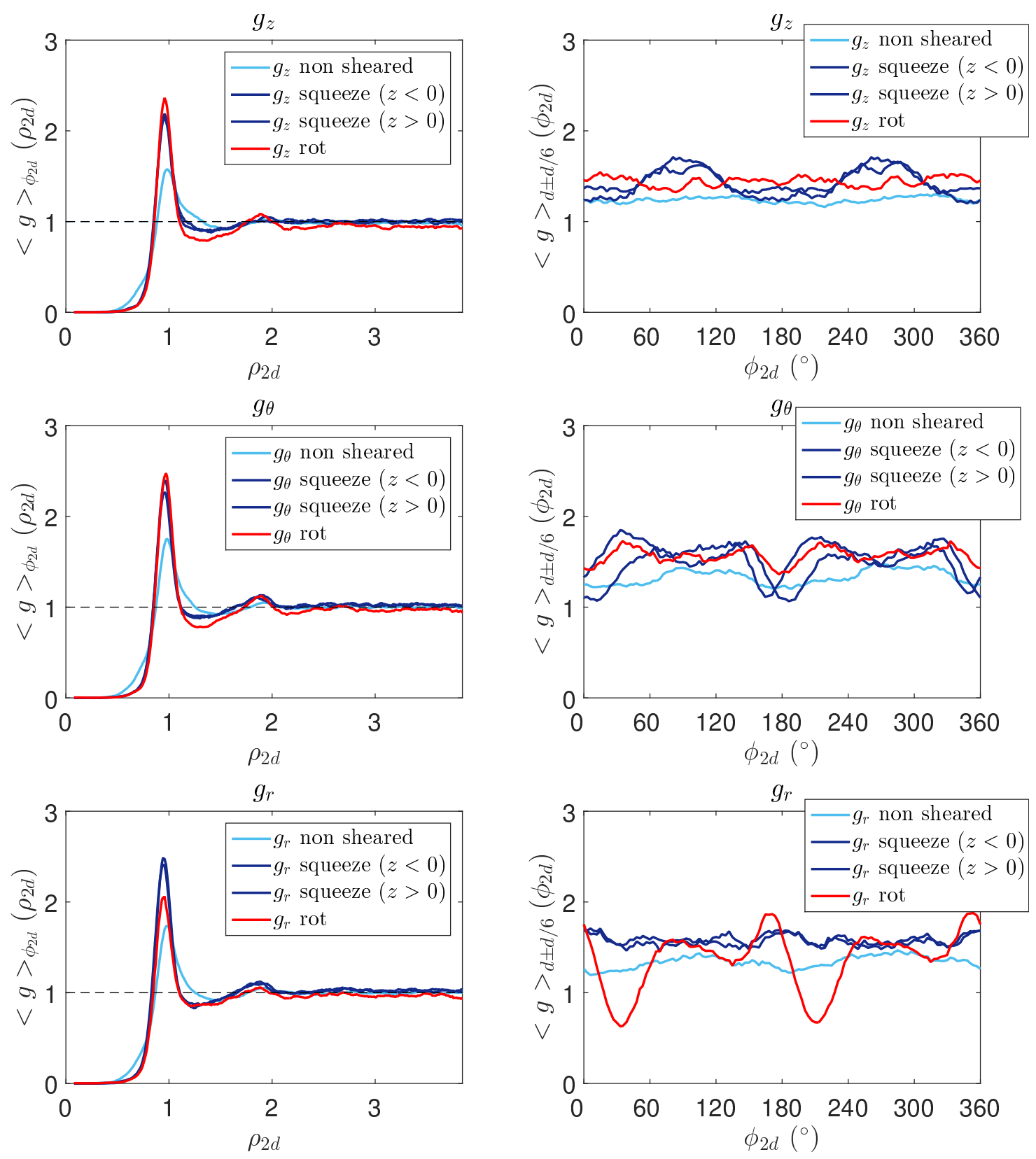

FIG. 19. Scalar pair distribution functions $<g\left(\rho_{2 d}\right)>_{\phi_{2 d}}$ averaged for all values of $\phi_{2 d}$ and $<g\left(\phi_{2 d}\right)>_{\rho_{2 d}=d \pm d / 6}$ averaged for distances $\rho_{2 d}=d \pm d / 6$ plotted as a function of the distance $\rho_{2 d}$ and the angle $\phi_{2 d}$ respectively in cartesian coordinates for a visco-plastic suspension at a solid fraction $\phi \simeq 35 \%$. In the first, second and third lines, $<g\left(\rho_{2 d}\right)>_{\phi_{2 d}}$ and $<g\left(\phi_{2 d}\right)>_{\rho_{2 d}=d \pm d / 6}$ are computed from $g_{z}, g_{\theta}$ and $g_{r}$ in the $(\theta r),(r z)$ and $(\theta z)$ planes respectively. Light blue lines correspond to a non sheared suspension, dark blue ones to a suspension sheared by a squeeze flow (in the two bottom and top half-planes $z<0$ and $z>0$ ) and red lines correspond to a suspension sheared by rotation. 
577 in the literature for non-Brownian suspensions, the flow-alignment of particle pairs was not, 578 as well as the presence of secondary maxima and minima. By contrast, a flow-alignment was 579 observed in numerical simulations of Brownian suspensions at small and moderate Peclet 580 numbers, even when which Brownian forces contribute preponderantly over hydrodynamics 581 force $[8,15-18]$.

${ }_{582}$ To our knowledge, our measurements of 3D microstructure of visco-plastic suspensions 583 are the first ones reported in the literature. We have previously presented some of them in 584 [5]; we recall briefly their main features in the next section.

585 D. Microstructure of the visco-plastic suspension at different shear rates

586 Unlike a Newtonian suspension, whose shear-induced microstructure does not depend on 587 the value of the shear rate for steady states [24], we could expect a different behaviour for a 588 visco-plastic suspension, due to its visco-plastic rheological behaviour. For comparison, the 589 microstructure of a Brownian suspension depends strongly on the shear rate through the 590 Peclet number (comparing hydrodynamic shear with thermal forces), as well as its rheology, 591 that exhibits both shear-thinning, Newtonian and shear-thickening behaviours [8, 15-18].

${ }_{592}$ Here, we compare the pdfs in the shear plane $(\theta z)$, where an anisotropic microstructure 593 develops when the suspension is rotationally sheared, for two different shear rates: $10^{-2} \mathrm{~S}^{-1}$ 594 and $10 \mathrm{~s}^{-1}$. For the visco-plastic fluid we used, this corresponds respectively to Bingham 595 numbers 1.02 and 1.62 (ratio of the shear stress over the yield stress) and to effective vis${ }_{596}$ cosities 51Pa.s and 1.6Pa.s (computed as $\left.\left(\tau-\tau_{y}\right) / \dot{\gamma}\right)$. At first order, in both cases, the 597 microstructure is anisotropic showing an extensional-depletion of particle pairs and a flow598 alignment of particle pairs. At second order, we do observe another kind of spatial variations 599 of $g$ and a fine difference for the two different shear rates: the depletion of particle pairs 600 in close contact is enhanced by high shear rates (Fig. 20), counter-balanced by a growing 601 tail of large values of $g$ appearing at a distance close to the particles diameter $(\xi \simeq d)$ from 602 the direction of the flow in the extensional stress domain (for $\ell \gtrsim d$ ). This tail is quantified 603 and compared for the two shear rates in Figure 20c, where two profiles of the pdf $g_{r}(\ell)$ at ${ }_{604}$ different positions $(\xi=+d$ and $\xi=-d)$ are drawn. These fine differences of microstructure 605 may have strong consequences on macroscopic rheology; more precisely, it was shown that 606 the shear stress at the onset of flow ("static" yield stress) strongly depends on the preshear 

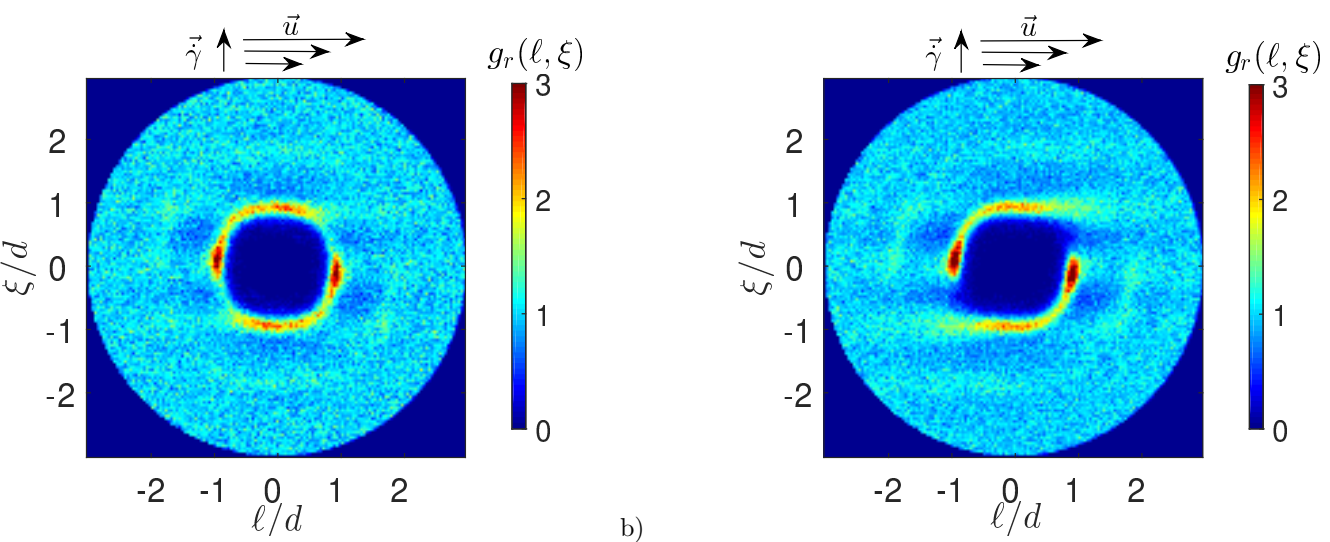

a)

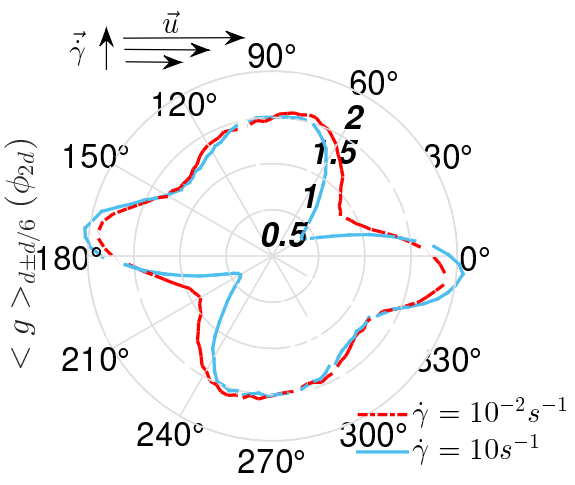

c) b)

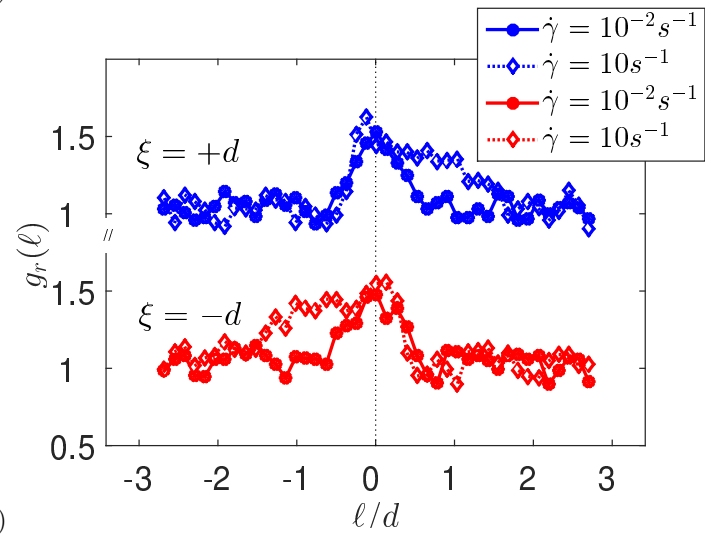

FIG. 20. a-b) Pdf in the shear plane $(\theta z)$ for a steady rotational shear of a visco-plastic suspension at a solid fraction $\phi \simeq 35 \%$ for a low $\left(\dot{\gamma}=10^{-2} \mathrm{~s}^{-1}\right.$, a) and a high $\left(\dot{\gamma}=10 \mathrm{~s}^{-1}\right.$, b) shear rate. c) Pdf averaged for $\rho_{2 d}=d \pm d / 6$ as a function of the angle $\phi_{2 d}$ in polar coordinates for the two shear rates. d) Some profiles of the pdf at different positions: $g_{r}(\ell)$ for $\xi=-d$ and $\xi=d$ for the two shear rates

607 history [5]. This was attributed to the difference in the microstructures jammed at rest after 608 the preshear. As a conclusion, the shear-thinning rheology of the suspending visco-plastic ${ }_{609}$ fluid, the presence of a yield stress or its elasticity may be responsible of the influence of the 610 shear rate on the microstructure and the related rheology.

\section{E. Bulk microstructure of a Newtonian suspension after a rotational shear flow}

${ }_{612}$ We now analyse the 3D microstructure of a Newtonian suspension in our parallel plates 613 geometry.

${ }_{614}$ It is already known from experiments and numerical simulations that an anisotropic 615 microstructure develops in sheared Newtonian suspensions [7, 10, 14]. However, in the 

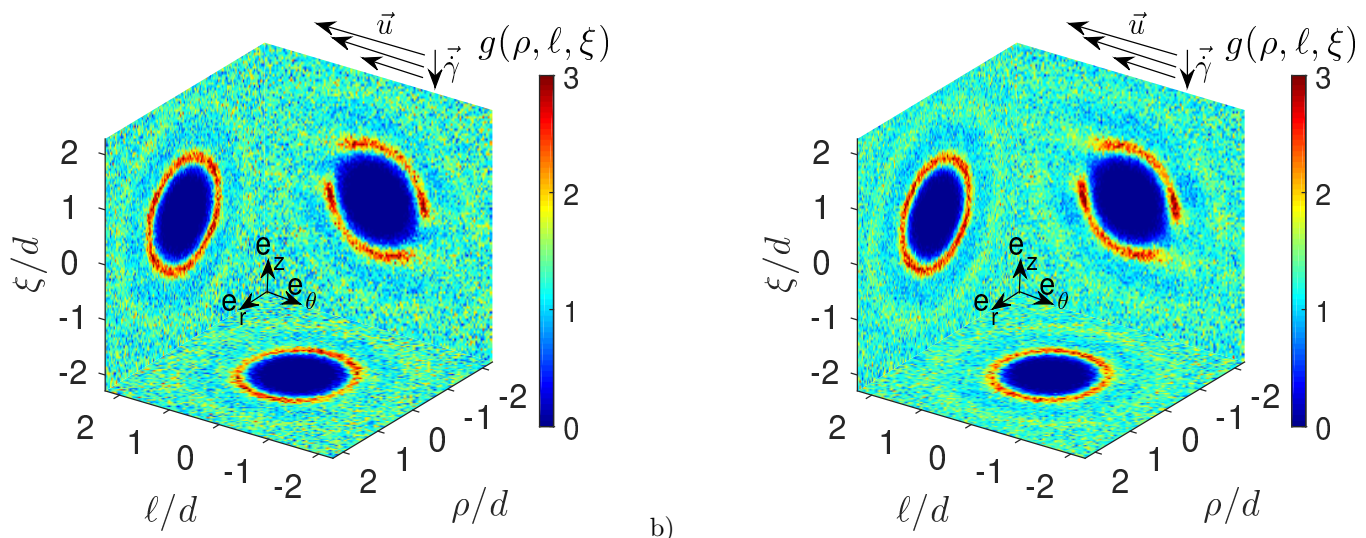

FIG. 21. 3D pair distribution functions in the shear plane in a parallel plates geometry for a Newtonian suspension of particles: for $\phi \simeq 36 \%$ (a) and $\phi \simeq 44 \%$ (b)

${ }_{616}$ literature, most pdfs are 2D, in the shear plane. The more reliable data were obtained ${ }_{617}$ in a Couette geometry, which presents a stress inhomogeneity in the shear plane, whereas ${ }_{618}$ the stress inhomogeneity in the parallel plates geometry we use is in the velocity gradient${ }_{619}$ vorticity $(z \theta)$ plane. Another difference between these two geometries is that shear stresses ${ }_{620}$ and shear rates are imposed in a Couette geometry and in a parallel plates one respectively. ${ }_{621}$ Endly, whereas the microstructure in $[10,14]$ is imaged under a flow, we are using a flow${ }_{622}$ arresting technique[18], so there may be some relaxation of the microstructure. In the ${ }_{623}$ following, we compare our 3D pdf obtained in our parallel plates set-up with 2D data ${ }_{624}$ obtained in a Couette geometry by Blan et al. [14] for the same Newtonian suspension.

${ }_{625}$ In the experiments presented here, we focus on steady simple shear flows controlled 626 by the rotation of the top plate of the parallel plates rheometer. Figure 21 shows the ${ }_{627}$ microstructure through pair distribution functions in the three cylindrical 'planes' $g_{r}(\ell, \xi)$, ${ }_{628} g_{\theta}(\rho, \xi)$ and $g_{z}(\rho, \ell)$ : it is not the same in the three planes. $g_{r}(\ell, \xi)$ becomes anisotropic in ${ }_{629}$ the shear plane, while it is still nearly isotropic in the two other 'planes'. Figure 21 shows 630 the microstructure of two Newtonian suspensions for two different solid fractions $\phi \simeq 36 \%$ ${ }_{631}$ and $\phi \simeq 44 \%$ : they are the same.

${ }_{632}$ We can show an extensional-depletion of particle pairs and a flow-alignment of particle ${ }_{633}$ pairs. Secondary maxima and minima are observed too here and (experimental and numeri634 cal) results from Blanc $[14,24]$ show that these two local maxima and minima are visible for ${ }_{635}$ solid fractions larger than $\phi \simeq 30 \%$. For comparison, Figure 22 reports two $2 \mathrm{D}$ pdfs mea${ }_{636}$ sured by Blanc et al for $\phi \simeq 35 \%$ and $\phi \simeq 45 \%$ for the same suspension but in a different 

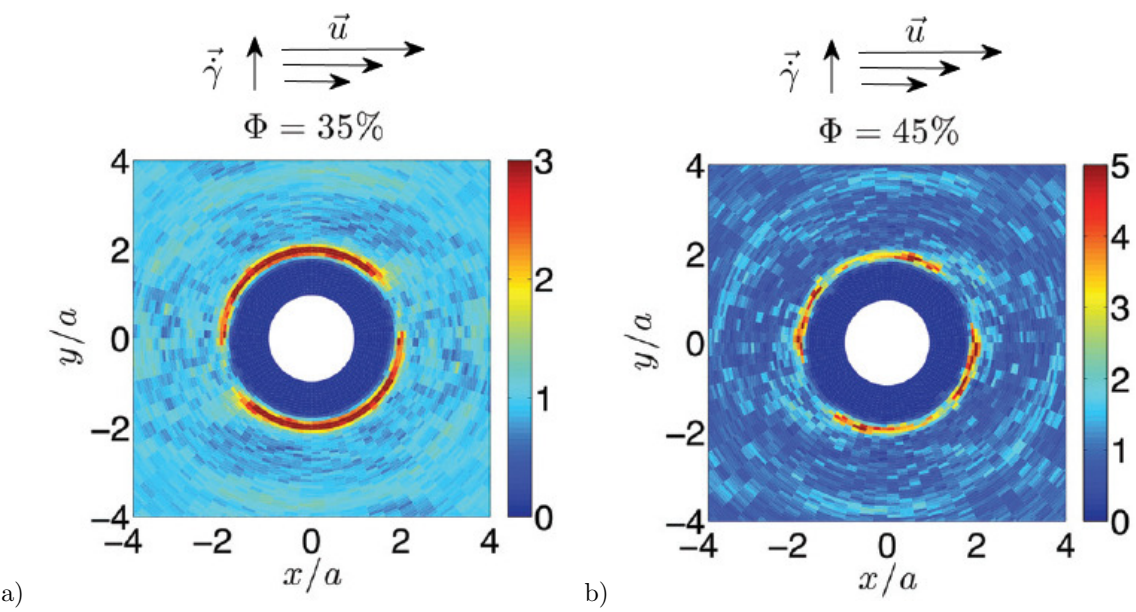

FIG. 22. Pair distribution functions in the shear plane from the literature (Blanc et al [14]) in a Couette geometry (co-centric cylinders) for a Newtonian suspension of particles: in the bulk for $\phi \simeq 35 \%$ (a) and $\phi \simeq 45 \%$ (b). Here $a$ is the particles radius, whereas $d$ is the diameter. Note the different color scales in a and b.
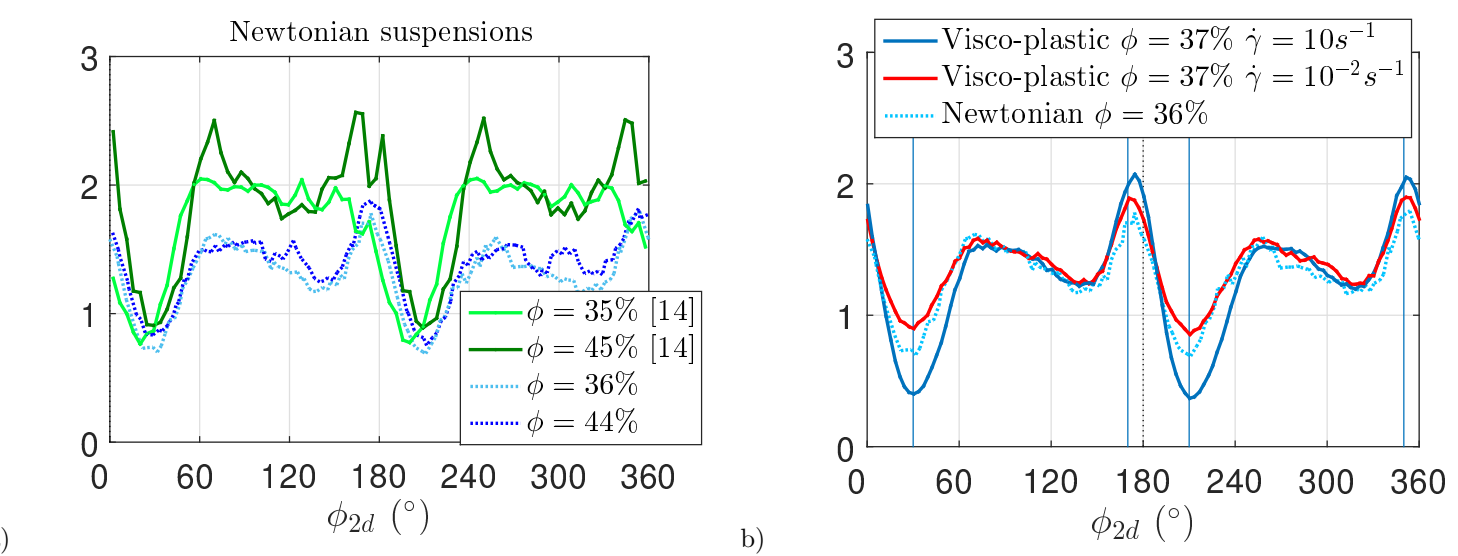

FIG. 23. Scalar angular pdf in the shear plane: (a) from Blanc et al in a Couette geometry for $\phi \simeq 35 \%$ and $\phi \simeq 45 \%$ and in our parallel plates geometry for $\phi \simeq 36 \%$ and $\phi \simeq 44 \%$ for the same Newtonian suspension; (b) in our parallel plates geometry for a Newtonian suspension at $\phi \simeq 36 \%$ and a visco-plastic suspension at $\phi \simeq 37 \%$ at a low $\left(10^{-2} s^{-1}\right)$ and high $\left(10 s^{-1}\right)$ shear rate

${ }_{637}$ geometry: they found that the angle of the minima $\theta_{\min }$ of $g$ changes from $21^{\circ}$ to $34^{\circ}$. More ${ }_{638}$ generally, they observed a strong influence of the solid fraction $\phi$ on $\theta_{\min }: 4.5^{\circ}$ for $\phi=5 \%$ 639 and $37^{\circ}$ for $\phi=56 \%$ [14].

${ }_{640}$ We now compare the values of $g\left(\rho_{2 d}, \phi_{2 d}\right)$ averaged close to the particle diameter $(\rho=$ $\left.{ }_{641} d \pm d / 6\right)$ as a function of $\phi_{2 d}$ in the shear plane for Newtonian suspensions and visco-plastic 
${ }_{642}$ suspensions in Figure 23. The maxima and minima described above are clearly visible in this ${ }_{643}$ representation. The shape and the position of the maxima/minima in the Blanc experiments 644 and in our experiment are in very good agreement. However, there are some quantitative ${ }_{645}$ differences. We checked that they are not due to the radial thickness $\Delta$ over which data are ${ }_{646}$ averaged nor to the width $\delta \rho=d / 6$ for the average $<g\left(\phi_{2 d}\right)>_{\rho_{2 d}=d \pm \delta \rho}$, neither the position ${ }_{647} R_{0}$ of the toroidal region where we compute our pdf. To this date, we cannot explain these 648 differences.

${ }_{649}$ We also note that the angular dependence of the pdf $<g\left(\phi_{2 d}\right)>_{\rho_{2 d}=d \pm \delta \rho}$ close to contact 650 of particles in visco-plastic fluids is very close to that in Newtonian suspensions. We note 651 however that the amplitude of the minimum and of the maximum change with the shear ${ }_{652}$ rate, which is specific to a nonlinear suspending fluid. It seems that more depletion can be 653 obtained in a visco-plastic fluid at a high shear rate than in a Newtonian fluid.

${ }_{654}$ As a conclusion, in our geometry the main difference of microstructure between Newto655 nian and visco-plastic suspensions is the dependence on the shear rate for visco-plastic and ${ }_{656}$ the independence on the shear rate for Newtonian. However, in all cases (visco-plastic or ${ }_{657}$ Newtonian, parallel plates or Couette geometry), the microstructure becomes anisotropic ${ }_{658}$ in the shear plane characterized by both an extensional-depletion of particle pairs and a 659 flow-alignment of particle pairs at similar angles. Finally, there may be a difference of quan660 titative values of the pdf (and of the microstructure) of a suspension in a Couette geometry ${ }_{661}$ and in a parallel plates one, where shear stresses and shear rates are imposed respectively 662 and where a stress inhomogeneity is in the shear plane and in the velocity gradient-vorticity ${ }_{663}(z \theta)$ plane respectively. Endly, whereas the microstructure in [14] is imaged under a flow, ${ }_{664}$ here we are using a flow-arresting technique[18], so there may be some relaxation of the 665 microstructure.

\section{${ }_{666} \quad$ F. Microstructure near solid walls: macroscopic layering}

${ }_{667}$ In our parallel plates geometry, the suspension is confined between two plates and the gap ${ }_{668}$ is not so large compared to the size of particles $(H / d \simeq 14$ for the visco-plastic suspension 669 and 12 for the Newtonian one) - this aspect ratio being common for such set-ups. These 670 solid boundaries induce some layering of particles, as shown by the solid fraction profiles 671 as a function of $z$ for smooth and rough surface plates (Fig. 24). Even when the surfaces 

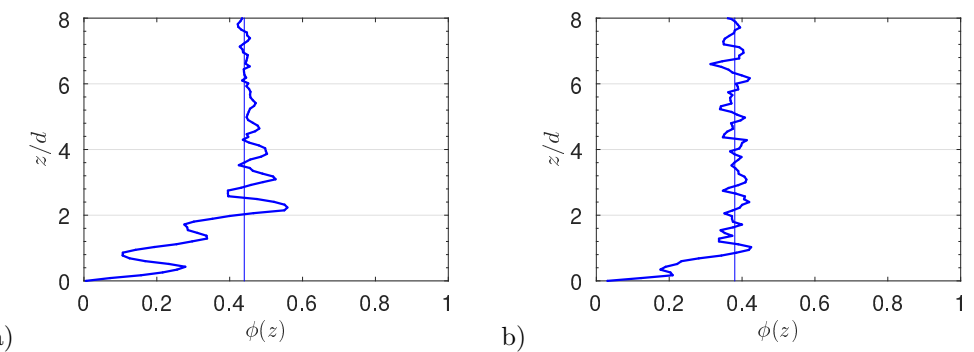

FIG. 24. Vertical concentration profiles close to a solid boundary for smooth (a) versus rough (b) surfaces after a rotational shear of $\approx 5 s^{-1}$ for a visco-plastic suspension at a solid fraction $\phi \simeq 40 \%$.

a)

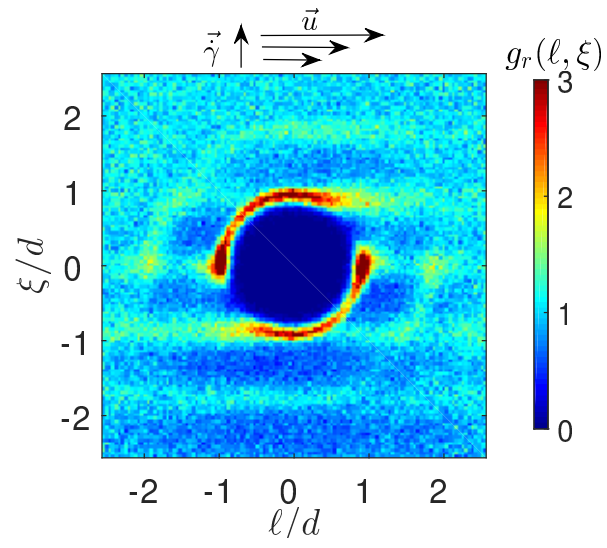

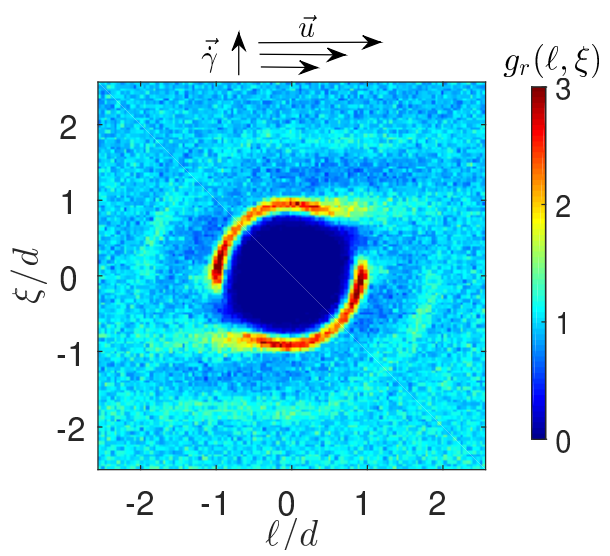

b)

FIG. 25. Pair distribution function $g_{r}$ in the shear plane $(\theta z)$ computed near a smooth solid wall located in the bottom (a) and in the bulk far from any solid wall (b).

672 have been roughened (Fig. 2c and 24b), we may observe a few alternate layers of high and 673 low solid fraction values, suggesting about 1 layer of aligned particles laying parallel to the 674 walls. In comparison with the case of smooth surfaces (Fig. 24a), the layering is reduced for 675 rough surfaces (Fig. 24b), and is localized close to the solid boundaries, whereas layering 676 comes deep inside the gap for smooth surfaces.

${ }_{677}$ This macroscopic layering close to solid boundaries is also visible on pair distribution 678 functions in the shear plane, when comparing pdfs computed close to solid boundaries with 679 pdfs computed in the bulk (far from any solid surface) for smooth solid plates (Fig. 25). A ${ }_{680}$ periodic pattern of strips is superimposed on the bulk microstructure. This effect was already 681 reported by $[24,35]$ for Newtonian suspensions, so this layering does exist for suspensions 682 of different rheology. 

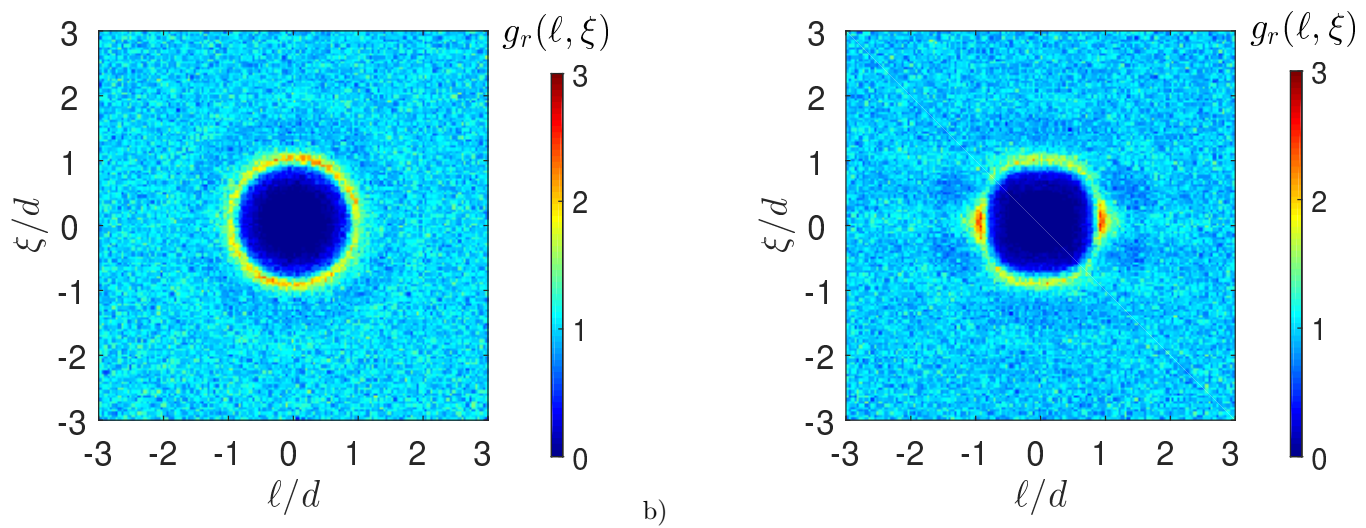

FIG. 26. Pair distribution function $g_{r}$ in the orthoradial plane $(\theta z)$ computed in the bulk (i.e. $R_{0}<0.60 D / 2,1.2 H<Z_{0}<2.2 H$ ) (a) and near the orthoradial free surface (i.e. $0.60 D / 2<R_{0}$, $\left.1.2 H<Z_{0}<2.2 H\right)(\mathrm{b})$ in the non sheared configuration (drop). The later pdf (b) is not observed anywhere else in the suspension than in the upper part of the suspension drop.
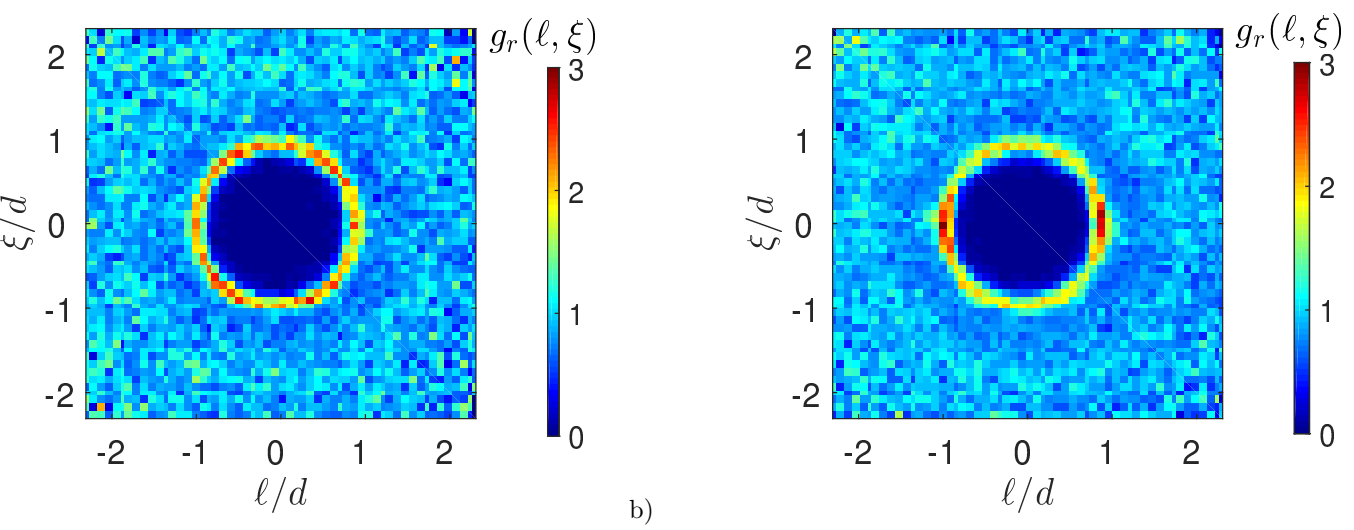

FIG. 27. a-b) Pair distribution function $g_{r}$ in the orthoradial plane $(\theta z)$ computed in the bulk (i.e. $R_{0}<0.60 D / 2,0.5 H / 2<Z_{0}$ ) (a) and near the orthoradial free surface (i.e. $0.60 D / 2<R_{0}$, $0.5 H / 2<Z_{0}$ ) (b) in the loaded and squeezed configuration. The later pdf (b) is not observed anywhere else in the suspension than in the quarter cylindrical part of the suspension near the top plate.

684 boundaries, even if they are rough. 

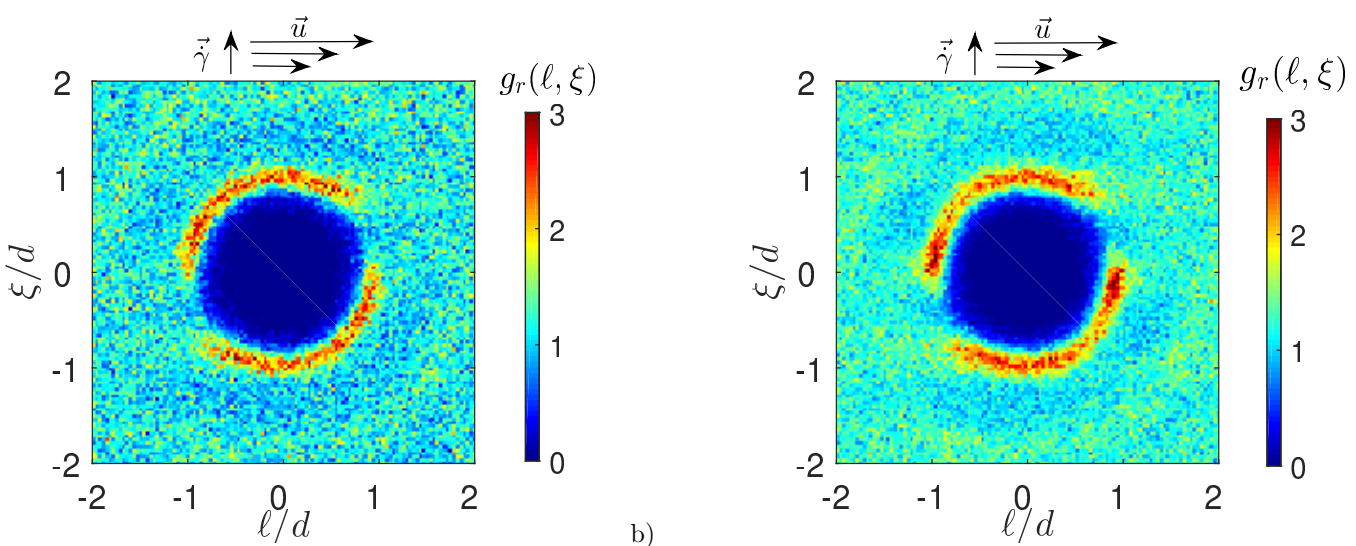

a)

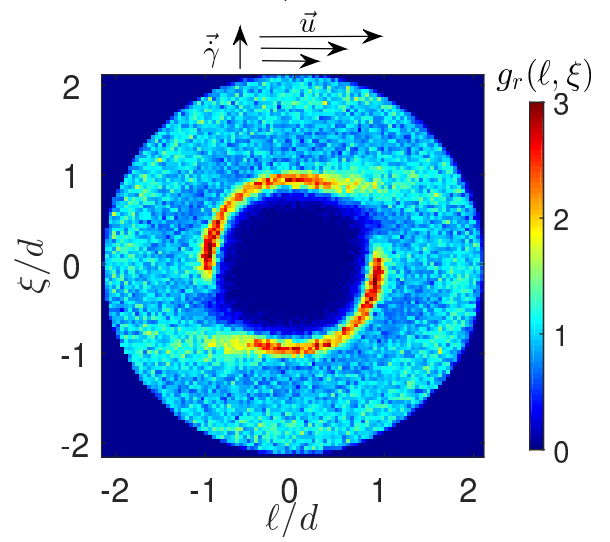

b)

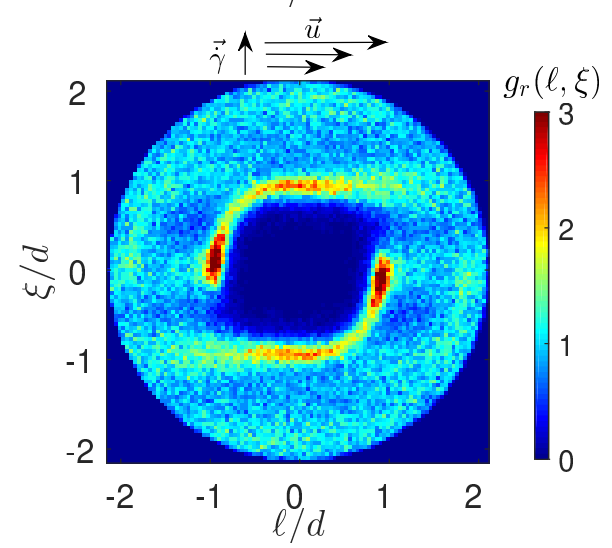

FIG. 28. Pair distribution function $g_{r}$ in the shear plane $(\theta z)$ far from (a, c) and close to (b, d) the orthoradial free interface for a Newtonian (a, b) and a visco-plastic suspension $(\mathrm{c}, \mathrm{d})$ 686 interfaces

687 A more subtle structuration is observed here near the orthoradial free interface, probably 688 enhanced when coupled with a shear flow (or with a flow in general). This structuration is 689 observed both in non sheared (drop) and sheared (by squeeze and rotation flows) suspensions 690 (Fig. 26, 27 and 28): this is an alignment of particle doublets (two particles in close contact) ${ }_{691}$ with the free interface along $\phi_{2 d} \simeq 0^{\circ}$ and $\phi_{2 d} \simeq 180^{\circ}$. However, whereas this alignment ${ }_{692}$ is systematically observed for rotational shear experiments, it is less pronounced and more ${ }_{693}$ localized in experiments of squeeze shear and in non loaded drop. This suggests that the ${ }_{694}$ occurrence of secondary flows in the squeeze flow (see part IV B) and when pouring the ${ }_{695}$ non loaded drop (see part IV A) may play a role in this structuration, due to a coupling 696 between free interfaces and flows. The pair distribution function exhibits a larger maxima 697 in the orthoradial plane $g_{r}(\ell, \xi)$ for values of $\ell \simeq d$ and $\xi \simeq 0$ when computed near the free 

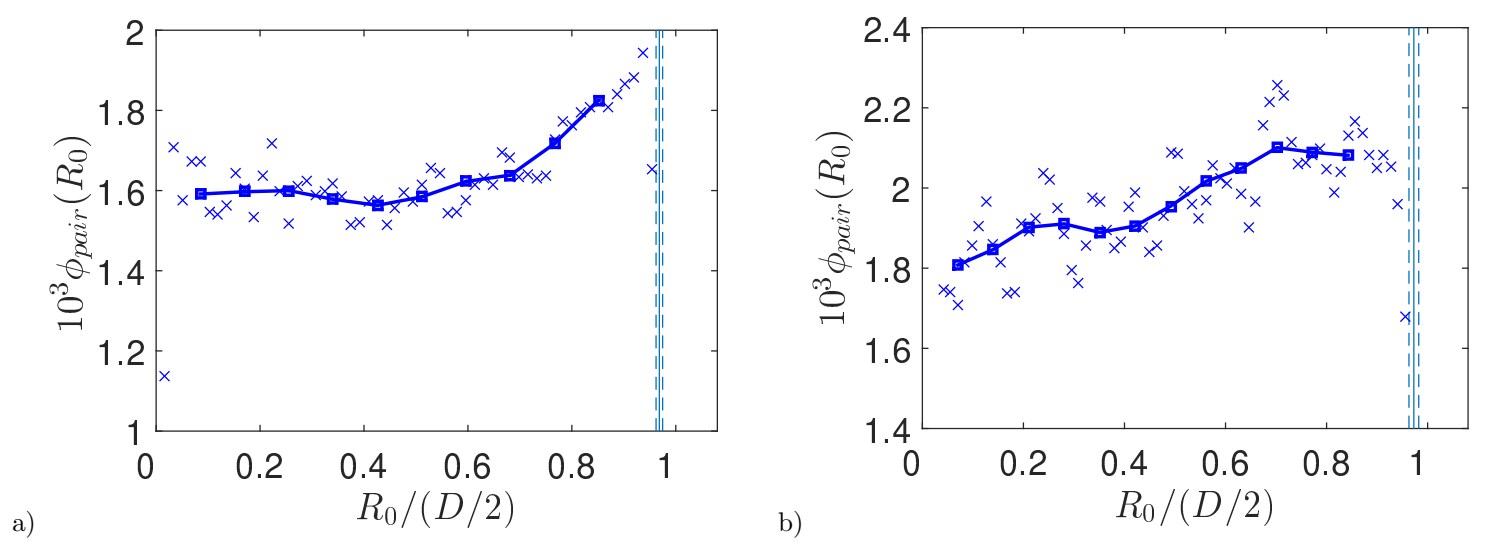

FIG. 29. Radial profiles of density (number per unit volume) of particle pairs aligned with the local flow $\left(\vec{e}_{\theta}\right) \phi_{\text {pair }}\left(R_{0}\right)$ for a Newtonian $(\phi \simeq 45 \%$, a) and a visco-plastic suspension $(\phi \simeq 38 \%$, b), for uniform radial profiles of solid fractions $\phi\left(R_{0}\right)$.

${ }_{698}$ interface (Fig. 26, 27 and 28), that is not visible on pdf computed in the bulk (far from the ${ }_{699}$ orthoradial free interface). This maxima of the pdf corresponds to an over-population of 700 particle doublets in close contact in the azimuthal (flow) direction; it is counter-balanced 701 by the apparition of a minima (drop, squeeze) or by a decrease of the secondary minima 702 (rotation), resulting in a modification of the shape of the pdf. This feature may recall the 703 flow-induced structural transition (from disorder to order to disorder as the Peclet number 704 is increased) observed in numerical simulations of Brownian suspensions [8, 15-18].

705 This can be observed at the macroscopic scale by computing $\phi_{\text {pair }}$, the density (number 706 per unit volume, normalized by the solid fraction) of particle doublets in close contact aligned 707 with $\vec{e}_{\theta}$ in toroidal regions at different radial positions $R_{0}$ (Fig. 29). This density is found 708 to increase close to the free interface in a region of width about $D / 8$ (Fig. 29), despite 709 some spatial fluctuations of $\phi_{\text {pair }}$ We may wonder if this local alignment is due to a steric 710 constraint of the free interface or to capillary stresses due to the contact lines between air, 711 particles and the fluid, and a coupling with solid walls or with some flows.

${ }_{712}$ We observe this specific microstructure near the free interface both for Newtonian and 713 for visco-plastic suspensions in a parallel plates geometry (Fig. 28, Fig. 29). Interestingly, 714 this allows us to provide an explanation for the observation of a peak in the flow direction 715 for the first experimental pdfs of Newtonian suspensions reported in the literature [10] 716 and reprinted here (Fig. 30), which has long been a puzzle. Indeed, pdfs from Parsi et 717 al. [10] were computed from optical observations of the first layer of particles on top of 


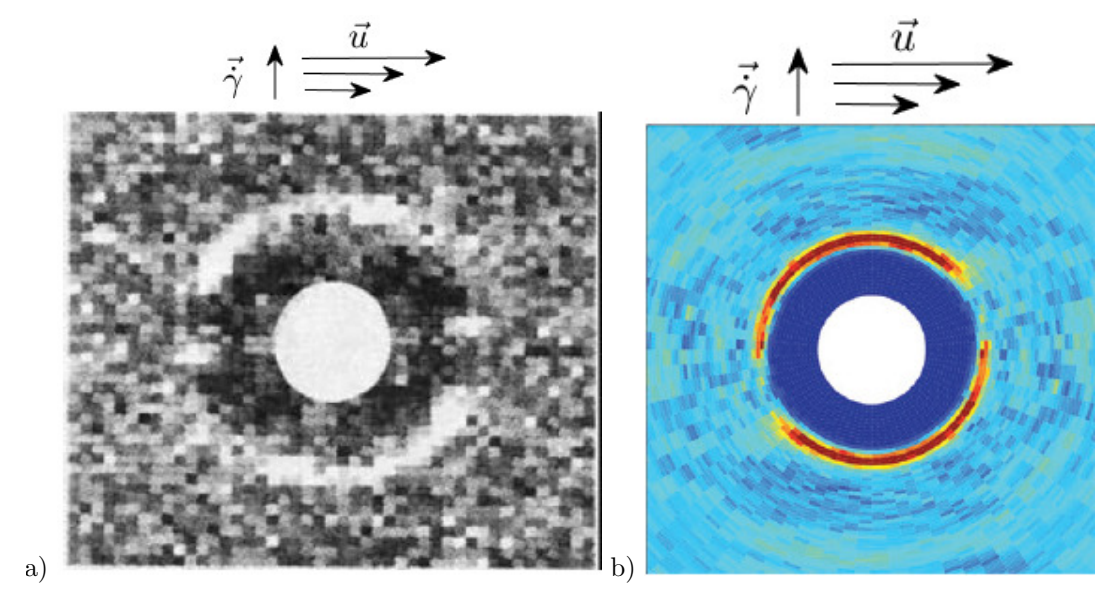

FIG. 30. Pair distribution functions from the literature in a Couette geometry (co-centric cylinders) for a Newtonian suspension of particles: a) close to the top free surface for $\phi=40 \%$ from [10], b) far from the top free surface for $\phi \simeq 35 \%$ from [14]; the top free surface being also the shear plane.

718 a Couette geometry (co-centric cylinders), that is from the observation of particles close 719 to the horizontal free interface, which is also the shear plane. Their results contrast with ${ }_{720}$ the pdfs reported by [14], computed from the particle positions in the bulk of a Couette ${ }_{721}$ flow and reprinted here (Fig. 30), in which such a peak is absent. The same conclusion 722 can thus be drawn from these studies (near and far from the free interface) as from our 723 observations: some peak appears in the pdf in the shear plane corresponding to an over724 population of particle doublets in close contact in the flow direction near the free surface 725 (Fig. 28, Fig. 30). Note that in both cases (parallel plates and Couette geometry) the free 726 surface is orthogonal to vorticity; no prediction can yet be made from our observations for 727 a free surface orthogonal to the velocity gradient, as in a flow along an inclined plane.

${ }_{728}$ As a conclusion, studying the bulk microstructure imposes to be far enough from both 729 free interfaces and solid boundaries; this precludes optical studies on non-index-matched 730 suspensions.

\section{${ }_{731}$ V. CONCLUSION}

732 In this paper, we present a technique we developed to image in 3D hard particle sus${ }_{733}$ pensions with X-ray tomography. It allows for the three dimensional characterization of 734 the microstructure in space - in different planes and at different positions in the set-up 
735 - from particle center positions identified at a sub-voxel resolution, thanks to home-made 736 algorithms for image processing. An accurate characterization of anisotropic microstructure 737 is possible, even for particle pairs in close contact.

${ }_{738}$ The dedicated set-ups used here for imaging have been built so that they can also be 739 used in a rheometer to perform rheological measurements. It is thus possible to correlate 740 the 3D-microstructures and the rheological properties for a same shear history (see [5] for 741 an example).

742 To illustrate the technique presented here, we apply it to two materials: a visco-plastic 743 suspension and a Newtonian suspension, meaning that our non-Brownian particles are sus744 pended in a visco-plastic and a Newtonian fluid respectively. The Newtonian suspension 745 is the same as used by Blanc et al. [14], but it is loaded here in a different geometry and 746 characterized in 3D, both in the bulk and near the interfaces.

${ }_{747}$ In principle, all the details of our technique are given so that it should be possible to 748 reproduce them. Our method should work for many suspensions (with a contrat of X-ray 749 between particles and the fluid), as soon as particles do not move during the time of a X-ray 750 scan. This may prevent us from imaging Brownian particles $(d \lesssim 1 \mu m)$ with a conventional ${ }_{751} \mathrm{X}$-ray tomograph but may demand a quicker X-ray source.

${ }_{752}$ After a rotational shear, we find approximately the same shear-induced anisotropic pair 753 distribution function (in the shear plane) in the bulk of the Newtonian suspension as in ${ }_{754}$ Blanc et al. [14]. Moreover, we find that it is nearly isotropic in the two other planes.

755 The visco-plastic suspension is characterized in different configurations. Its microstruc756 ture is found to be nearly isotropic in 3D after preparation, showing that it is possible to ${ }_{757}$ design such a nearly isotropic suspension. It becomes anisotropic in the shear plane what${ }_{758}$ ever the shear is (rotational shear or continuous squeeze); loading in any geometry (except 759 the vane-in-cup) thus leads to an anisotropic initial state. The shear-induced pair distribu760 tion function for the visco-plastic suspension is found to be similar to that for a Newtonian ${ }_{761}$ suspension, except at high shear rates. Indeed, as a consequence of the non-linear nature of 762 the visco-plastic fluid (either the yield-stress or the shear-thinning viscosity), the shear rate 763 changes the anisotropy of the microstructure.

${ }_{764}$ For the two hard non-Brownian particles suspensions used here (Newtonian and visco765 plastic suspensions), we find some layering close to solid walls, especially if they are smooth, 766 and a more surprising layering close to the free interface. This latter is not as obvious as 


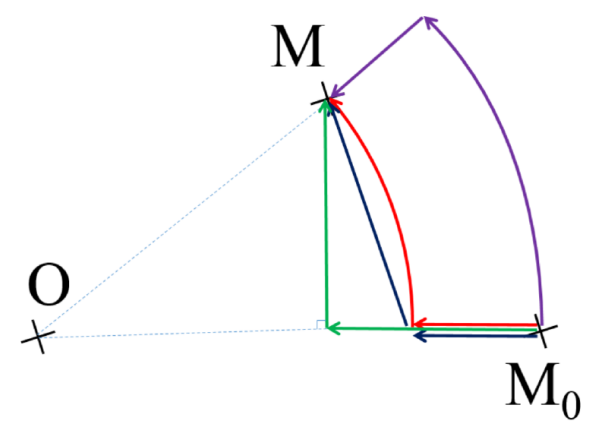

FIG. 31. Two particles $M$ and $M_{0}$ in the global cylindrical framework and four examples for the definition of the pair vector $\vec{r} \equiv \overrightarrow{M_{0} M}$ characterizing their separation: euclidean $\left(\vec{r}=\overrightarrow{M_{0} M}\right)$ or curvilinear - along circular flow lines - coordinates, with orthogonal or not orthogonal axis

767 the former because it is not strikingly visible on macroscopic quantities. It is visible at the 768 scale of some particle pairs only: it induces a flow-alignment of particle doublets at close ${ }_{769}$ contact, in regions of quite large extent close to free interfaces. This effect is likely to be a 770 consequence of a coupling between free interfaces and shear; it allows to explain previous 771 data of the literature [10].

\section{Appendix A: Possible geometrical choices to compute pair distribution functions}

773 Pair distribution functions are computed as normalized probabilities of finding a test 774 particle $M$ located at $(r, \theta, z)$ from another reference particle $M_{0}$ located at $\left(r_{0}, \theta_{0}, z_{0}\right)$ in the 775 cylindrical framework attached to the axis of the rheometer circular plates (see Part IIIC). 776 There are several choices for the characterization of the pair vector $\vec{r}$ characterizing their 777 separation: euclidean $\left(\vec{r}=\overrightarrow{M_{0} M}\right)$ or curvilinear (along circular flow lines) coordinates, 778 orthogonal or not orthogonal axis (unit vectors dependent on $M_{0}$ only or both on $M_{0}$ and $779)$. Four examples of possible definitions are shown in Figure $31:\left(r \cos \left(\left(\theta-\theta_{0}\right) / 2\right)-r_{0}\right.$, $\left.780 r \sin \left(\theta-\theta_{0}\right), z-z_{0}\right),\left(r-r_{0}, 2 r \sin \left(\left(\theta-\theta_{0}\right) / 2\right), z-z_{0}\right),\left(r-r_{0}, r_{0}\left(\theta-\theta_{0}\right), z-z_{0}\right)$ and ${ }_{781}\left(r-r_{0}, r\left(\theta-\theta_{0}\right), z-z_{0}\right)$. Euclidean coordinates can in principle be chosen but we believe 782 that non euclidean coordinates, but curvilinear coordinates projected along the cylindrical 783 'planes' aligned with the circular flow streamlines, are more relevant to characterize the 784 microstructure induced by a simple shear flow. We choose for coordinates of the pair vector ${ }_{785} \vec{r}=(\rho, \ell, \xi): \rho=r-r_{0}, \ell=r\left(\theta-\theta_{0}\right), \xi=z-z_{0}$. This choice does not induce any bias in 786 the symmetry of the pair distribution functions $g_{z}$, as well as of $g_{\theta}$ or $g_{r}$, as shown by the 

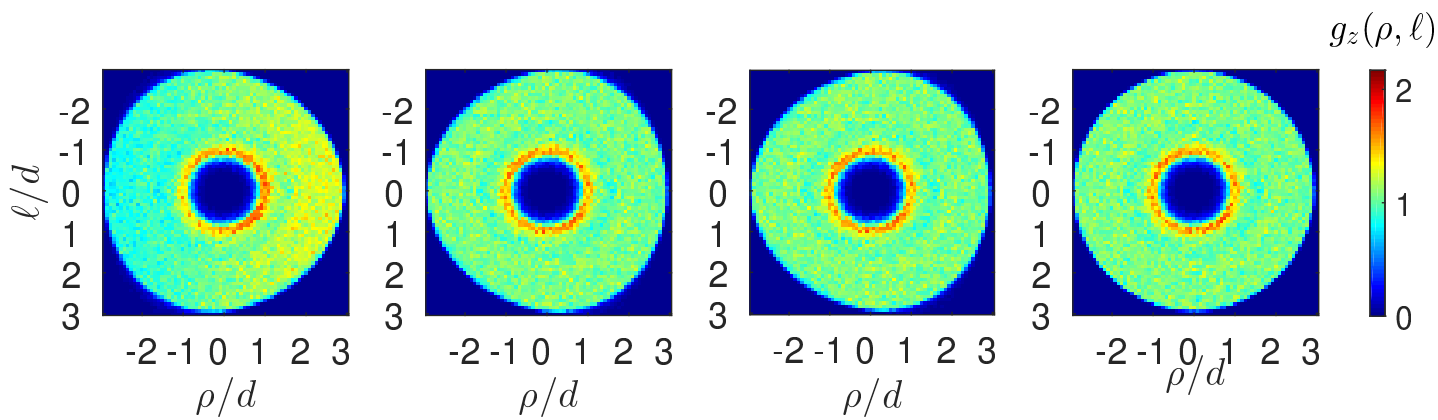

a)
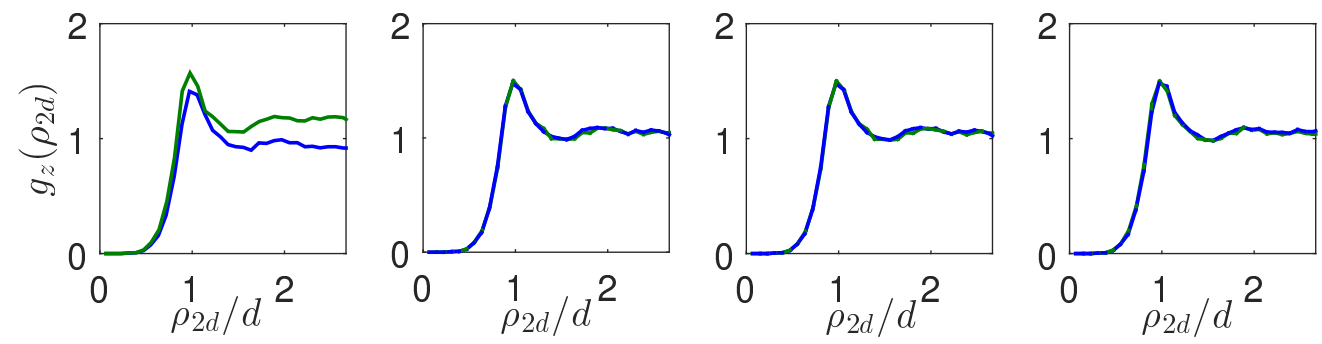

FIG. 32. Influence on the pair distribution function $g_{z}$ computed in $(\theta r)$ planes (in 2D (a), and in $1 \mathrm{D}(\mathrm{b}))$ of the definition of the pair vector $\vec{r}$ (for the four definitions written in the text). Our choice, Eq. (10) does not induce any bias in the symmetry of the pair distribution functions between negative and positive values of $\rho$

787 comparison in Figure 32 of $g_{z}$ computed in $(\theta r)$ planes for reference particles in a toroidal 788 ring at a small radius for the four previous different definitions of $\vec{r}$.

789 In order to get enough statistics, we need to define a thickness for the elementary planes 790 where we compute 2D pair distribution functions (see Part III C). Whereas the definition of 791 the thicknesses $\Delta z$ of horizontal planes $(\theta r)$ and $\Delta r$ of orthoradial 'planes' $(\theta z)$ is obvious, 792 the thickness of radial planes $(r z)$ offers several choices. Different possible choices for the 793 thickness of radial planes $(r z)$ are: constant euclidean thickness $r \sin \left(\theta-\theta_{0}\right)$, constant wedge 794 angle $\theta-\theta_{0}$, or constant arc length $r\left(\theta-\theta_{0}\right)$. Figure 33 shows the pair distribution function ${ }_{795} g_{\theta}$ computed in $(r z)$ planes (in 2D and in 1D) for the three previous different definitions 796 of the thickness. Whereas the definitions of a constant wedge angle and of a constant arc 797 length show a bias (there is an asymmetry between the left and right sides), the definition of 798 a constant euclidean thickness does not. That is why we choose to work with radial planes $799(r z)$ of thickness $\Delta h=r \sin \left(\theta-\theta_{0}\right)$. 
a)
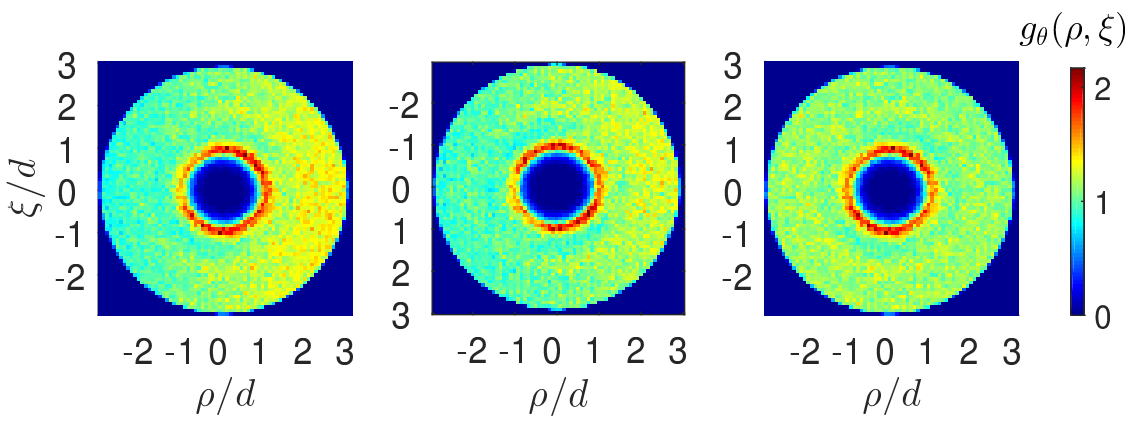

b)
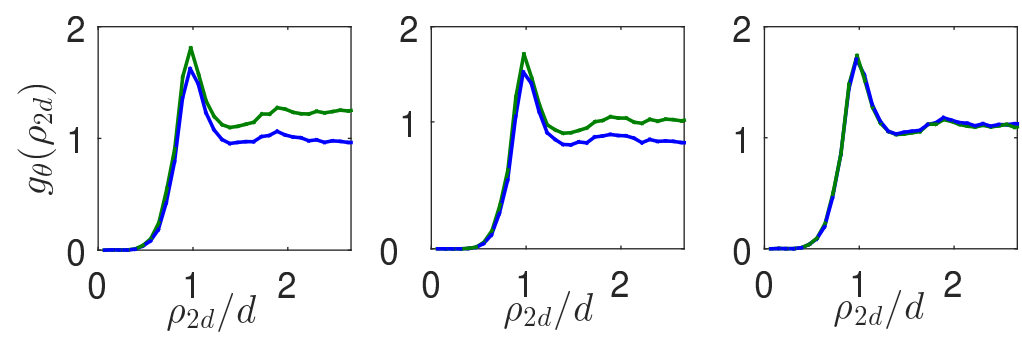

FIG. 33. Influence on the pair distribution function $g_{\theta}$ computed in radial $(r z)$ planes (in 2D (a) and in 1D (b)) of the definition of their thicknesses (for the three different definitions written in the text). The choice of a constant euclidean thickness $\Delta h=r \sin \left(\theta-\theta_{0}\right)$, Eq. 9 , does not induce any bias in the symmetry of the pair distribution functions between negative and positive values of $\rho$

\section{$800 \quad$ Appendix B: Statistical precision of pair distribution functions}

801 Here we discuss the statistics in terms of number of particles required for fine sampling and 802 good precision of the pdf $g$ from its following expression (see Part III C), where $N^{\text {pair }}(\vec{r} \pm \delta \vec{r})$ 803 is the number of pairs of particles separated by $\vec{r} \pm \delta \vec{r}$ and $N_{\text {ref }}$ is the total number of the 804 reference particles:

$$
g(\vec{r} \pm \delta \vec{r})=N^{\text {pair }}(\vec{r} \pm \delta \vec{r}) / N_{\infty}
$$

805 with:

$$
N_{\infty}=N_{\text {ref }} n_{0 v} \delta V
$$

806 the number of pairs separated by a vector $\vec{r}$ such that $\|\vec{r}\| \rightarrow \infty$ in the sampling volume $\delta V$ 807 in the space of pair vectors delimited by $\vec{r} \pm \delta \vec{r}$. Note that $n_{0 v} \delta V$ is the number of particles 808 in the sampling volume $\delta V$. The precision $\Delta g$ on $g(\vec{r})$, or the minimal detected variation, 809 corresponding to the variation of the integer $\Delta N^{\text {pair }}=1$, is:

$$
\Delta g=1 / N_{\infty}=\left(N_{\text {ref }} n_{0 v} \delta V\right)^{-1}
$$



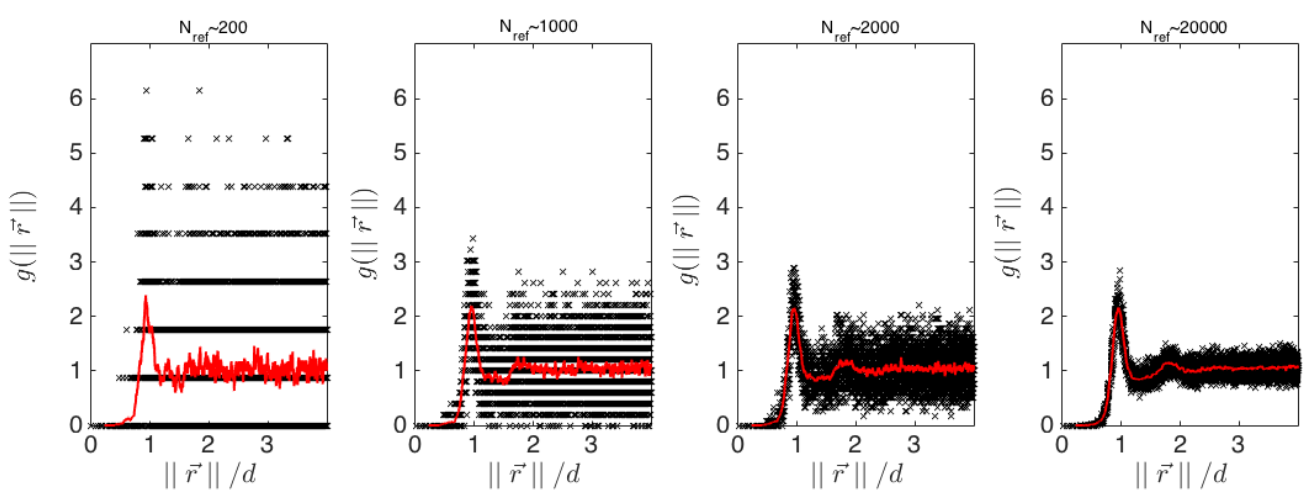

FIG. 34. Scalar pair distribution function $g(\|\vec{r}\|)$ for different numbers of reference particles $N_{\text {ref }}$ increasing from arbitrary small values to larger values (but for constant numbers of reference particles with $\|\vec{r}\|$, i.e. non adaptative spheres).

810 We will usually compute the pdf on toroidal rings of thicknesses $\Delta R$ and $\Delta Z$ centered s11 on $R_{0}$ and $Z_{0}$ (of volume $\vartheta=2 \pi R_{0} \Delta R \Delta Z$ ), so that the number of reference particles is 812 approximately:

$$
N_{r e f} \simeq n_{0 v} \vartheta
$$

${ }_{813}$ For a given density $n_{0 v}$ and chosen precisions on the spatial resolution $\delta V$ and on the pdf ${ }_{814} \Delta g$, this prescribes a minimum number of reference particles from Eq. (B3):

$$
N_{\text {ref }}=\left(n_{0 v} \delta V \Delta g\right)^{-1}
$$

815 or equivalently a minimum volume $\vartheta$ for the toroidal ring from Eq. (B4).

816 As a numerical example, if we want a precision $\Delta g=10^{-2}$ on $g(\vec{r})$ and a spatial resolution ${ }_{817}$ for the $2 \mathrm{D} \operatorname{pdf} \delta V=\Delta \delta^{2}$ with $\Delta=\mathrm{d}=140 \mu \mathrm{m}=12 \mathrm{vx}$ and $\delta=1 \mathrm{vx}=12 \mu \mathrm{m}$, for a solid ${ }_{818}$ fraction $\phi=35 \%$ and $\mathrm{d}=140 \mu \mathrm{m}\left(n_{0 v}=244 \mathrm{~mm}^{-3}\right)$, we need at least $2.10^{4}$ reference 819 particles in the region of interest. Otherwise, we may see the discrete nature of variations 820 of $g(\|\vec{r}\|)$ (Fig. 34). This effect of discretization may appear anyway for large inter-particle 821 distances, because of the use of adaptative spheres. As a compromise, these spatial averages 822 may lead to some underestimate of peak values of $g$, whether there are large variations of $g$.

\section{ACKNOWLEDGMENTS}

${ }_{824}$ This work was supported by a grant from Agence National de la Recherche (ANR 2010 825 JCJC 0905 01-SUSPASEUIL). The Laboratoire Navier microtomograph has been acquired 
826 ${ }_{827}$ Française de Mécanique).

[1] Thrane L. N., L. F. Nielsen, M. Brandl, and M. R. Geiker. On the effect of coarse aggregate fraction and shape on the rheological properties of self-compacting concrete. Cem. Concr. Aggregates, 24(1):3-6, 2002.

[2] Mahaut F., X. Chateau, P. Coussot, and G. Ovarlez. Yield stress and elastic modulus of suspensions of noncolloidal particles in yield stress fluids. J. Rheol., 52(1):287-313, 2008.

[3] Denn M. M. and J. F. Morris. Rheology of non-brownian suspensions. Annual Review of Chemical and Biomolecular Engineering, 5:203-228, 2014.

[4] Chateau X., G. Ovarlez, and K. L. Trung. Homogenization approach to the behavior of suspensions of noncolloidal particles in yield stress fluids. J. Rheol., 52(2):489-506, 2008.

[5] Ovarlez G., F. Mahaut, S. Deboeuf, N. Lenoir, S. Hormozi, and X. Chateau. Flows of suspensions of particles in yield stress fluids. J. Rheol., 59:1449-1486, 2015.

[6] Hafid H., G. Ovarlez, F. Toussaint, P.H. Jezequel, and N. Roussel. Effect of particle morphological parameters on sand grains packing properties and rheology of model mortars. Cem. Concr. Res., 80:44-51, 2016.

[7] Morris J. A review of microstructure in concentrated suspensions and its implications for rheology and bulk flow. Rheol. Acta, 48:909923, 2009.

[8] Nazockdast E. and J. F. Morris. Microstructural theory and the rheology of concentrated colloidal suspensions. J. Fluid Mech., 713:420-452, 2012.

[9] Gadala-Maria F. and A. Acrivos. Shear-induced structure in a concentrated suspension of solid spheres. J. Rheol., 24(6):799-814, 1980.

48 [10] Parsi F. and F. Gadala-Maria. Fore-and-aft asymmetry in a concentrated suspension of solid spheres. J. Rheol., 31(8):725-732, 1987.

50 [11] Rampall I., J. R. Smart, and D. T. Leighton. The influence of surface roughness on the particlepair distribution function of dilute suspensions of non-colloidal spheres in simple shear flow. J. Fluid Mech., 339:1-24, 1997.

[12] Blanc F., F. Peters, and E. Lemaire. Experimental signature of the pair-trajectories of rough spheres in the shear-induced microstructure in non-colloidal suspensions. Phys. Rev. Lett., 
856 [13] Blanc F., F. Peters, and E. Lemaire. Local transient rheological behavior of concentrated $857 \quad$ suspensions. J. Rheol., 55(4):835-854, 2011.

858 [14] Blanc F., E. Lemaire, A. Meunier, and F. Peters. Microstructure in sheared non-brownian

860 [15] Phung T. N., J. F. Brady, and G. Bossis. Stokesian dynamics simulation of brownian suspen$861 \quad$ sions. J. Fluid Mech., 313:181-207, 1996.

862 [16] Foss D. R. and J. F. Brady. Structure, diffusion and rheology of brownian suspensions by stokesian dynamics simulation. J. Fluid Mech., 407:167200, 2000.

864 [17] Morris J. F.and B. Katyal. Microstructure from simulated brownian suspension flows at large shear rate. Phys. Fluids, 14(1920), 2002.

866 [18] Gao C., S. D. Kulkarni, J. F. Morris, and J. F. Gilchrist. Direct investigation of anisotropic

870 [20] Goyon J., A. Colin, G. Ovarlez, A. Ajdari, and L. Bocquet. Spatial cooperativity in soft glassy $871 \quad$ flows. Nature, 454(7200):84-87, 2008.

872 [21] Maire E., J. Y. Buffiere, L. Salvo, J. J. Blandin, W. Ludwig, and J. M. Letang. On the

875 [22] Hsieh J. Computed tomography: principles, design, artifacts, and recent advances. Wiley

877 [23] Heindel T. J. A review of x-ray flow visualization with applications to multiphase flows.

879 [24] Blanc F. Rhéologie et microstructure des suspensions concentrées non browniennes. $\mathrm{PhD}$

881 [25] Feldkamp L.A., L.C. Davis, and J.W. Kress. Practical cone-beam algorithm. JOSA A, $882 \quad 1(6): 612-619,1984$

883 [26] Amon A., P. Born, K. E. Daniels, J. A. Dijksma, K. Huang, D. Parker, M. Schroter, R. Stanapplication of x-ray microtomography in the field of materials science. Adv. Eng. Mater., 3:539546, 2001. Interscience, Bellingham Washington USA, 2009. Journal of Fluids Engineering, 133(7):074001, 2011. thesis, Université Nice Sophia Antipolis, 2011.

narius, and A. Wierschem. Preface: Focus on imaging methods in granular physics. Review of Scientific Instruments, 88(051701), 2017. 
${ }_{886}[27]$ Ni W.J. and H. Capart. Crosssectional imaging of refractiveindexmatched liquidgranular flows. ${ }_{887} \quad$ Exp. Fluids, 56:163, 2015.

${ }_{888}$ [28] Dijksman J. A., F. Rietz, K. A. Lorincz, M. van Hecke, and W. Losert. Invited article: Refrac-

889

890

891 [29] Gao Y. and Kilfoil M. L. Accurate detection and complete tracking of large populations of 892 features in three dimensions. Optics Express, 17(4685), 2009.

893 [30] Macosko C. W. Rheology: principles, measurements, and applications. Wiley-VCH, New York, $894 \quad 1994$.

895 [31] Ovarlez G., Q. Barral, and P. Coussot. Three-dimensional jamming and flows of soft glassy

896 materials. Nature Materials, 9(2):115-119, 2010.

897 [32] Ramachandran A. and D. T Jr Leighton. Particle migration in concentrated suspensions $898 \quad$ undergoing squeeze flow. J. Rheol., 54(3):563-589, 2010.

899 [33] Rueckel J., M. Stockmar, F. Pfeiffer, and J. Herzen. Spatial resolution characterization of a $900 \quad$ x-ray microct system. Applied Radiation and Isotopes, 94:230-234, 2014.

901 [34] More and more iterations are needed for convergence at higher solid fractions, but convergence 902

904 [35] Yeo K. and M. R. Maxey. Ordering transition of non-brownian suspensions in confined steady 905 shear flow. Phys. Rev. E, 81:051502, 2010. 